Bangladesh J. Plant Taxon. 22(1): 17-41, 2015 (June)

(C) 2015 Bangladesh Association of Plant Taxonomists

\title{
AN ANNOTATED CHECKLIST OF THE VASCULAR PLANTS OF SUNDARBAN MANGROVE FOREST OF BANGLADESH
}

\author{
Mohammad Sayedur Rahman ${ }^{1}$, Gazi Mosharof Hossain, \\ Saleh Ahammad Khan and SARder Nasir UdDin ${ }^{2}$ \\ Department of Botany, Jahangirnagar University, Savar, Dhaka 1342, Bangladesh
}

Keywords: Mangrove; Sundarban; Vascular plants.

\begin{abstract}
The study revealed the occurrence of 528 species of vascular plants belonging to 356 genera and 111 families in the Sundarban Mangrove Forest of Bangladesh. Among these species, 24 were pteridophytes and the rest were angiosperms, of which only 24 were true mangroves and 70 were mangrove associates. Magnoliopsida and Liliopsida were represented by 373 and 131 species, respectively. These species belonged to 345 herbs, 89 shrubs and 94 trees. Sixty-four species were climbers, 14 were epiphytes, 6 were parasites, and 7 were palms. The species number per family varied from 1 to 42 . In pteridophytes, Pteridaceae with 4 genera and 5 species was the largest family. In angiosperms, Fabaceae with 24 genera and 42 species and Poaceae with 27 genera and 42 species were the largest families, respectively, in Magnoliopsida and Liliopsida. Most of the species included in this checklist were found in oligohaline zone, Sarankhola range and the forest margins, and recognized as economically important. Eleven species categorized as threatened in Bangladesh were found to occur in this mangrove forest.
\end{abstract}

\section{Introduction}

The Sundarban, located in south of the Tropic of Cancer at the northern limits of the Bay of Bengal and covering a vast area of about 10,029 sq km in the territory of Bangladesh and India (Hussain and Acharya, 1994), is the world's largest single chunk of productive mangrove forest ecosystems (Das and Siddiqi, 1985). The major part (62\%) of this mangrove forest belongs to Bangladesh. Sundarban Mangrove Forest of Bangladesh (SMFB) is remarkably important for its floristic and faunal composition, wildlife habitats, and ecological values due to which it was declared as the world's 560th Ramsar Wetlands Site in 1992 and the 798th World Heritage Site by the UNESCO in 1999 (Nishorgo, 2008; Hossain et al., 2015).

The Sundarban is a unique ecosystem in the world (Biswas et al., 2007) and one of the biodiversity rich sites in Bangladesh (Prain, 1903). The forest of Bangladesh Sundarban is serving as a natural barrier against different natural calamities, like storms, cyclones and tidal bores for a large part of coastal settlements. The SMFB has drawn attention of the people through different socio-economic contributions to this country, especially supporting many industries, local economy and communities by supplying various raw materials, and its fantastic natural beauty. Floristic composition of this mangrove forest is considered as very rich compared to other mangroves in the world due to its geo-physical location near the Indo-Burma global prime hotspot region (Mittermeier et al., 1998).

Before and after Prain (1903), nobody, however, is known to conduct a detailed floristic study on the vascular plants surveying most of the areas of this mangrove that harbours diverse wildlife and parts of which are usually inaccessible due to very adverse weather in summer and monsoon.

${ }^{1}$ Corresponding author. Email: sayedur27bcs@gmail.com

${ }^{2}$ Bangladesh National Herbarium, Chiriakhana Road, Mirpur 1, Dhaka 1216, Bangladesh 
Hooker (1872-1897) studied the flora of the then British India including many areas that belong to the present political boundary of Bangladesh. Prain (1903) for the first time studied the flora of both Indian and Bangladesh Sundarban concurrently. After that, a small part of the flora or plant diversity of this mangrove was inventoried sporadically without providing information on plant specimens examined (e.g. Chapman, 1976; Chaffey et al., 1985; Das and Siddiqi, 1985; Karim, 1994; Rashid et al., 2008; Islam et al., 2014). Rahman et al. (2012, 2014) and Hossain et al. (2015) studied particular plant groups of this mangrove providing the lists of specimens examined.

Since no complete floristic study was carried out throughout the SMFB after Prain (1903), it is completely unknown if any change in the floristic composition of this mangrove has occurred over the last more than one hundred years. On the other hand, the SMFB belongs to one of the world's most risk-prone areas where the plant diversity is increasingly being depleted due to overextraction and destruction of natural resources, and on-going and predicted adverse impacts of climate change (Hussain, 2014). Therefore, there is a great need for conducting a detail floristic study throughout this mangrove forest. In the present study, an annotated checklist of vascular plant species of the SMFB has been constructed based on a thorough taxonomic inventory conducted throughout the area and examination of the representative plant specimens to provide the updated information on the floristic composition and distribution in the SMFB and a way of focusing and stimulating further work in this mangrove forest.

\section{Materials and Methods}

The SMFB lies between the latitudes $21^{\circ} 30^{\prime} \mathrm{N}$ and $22^{\circ} 30^{\prime} \mathrm{N}$ and longitudes $89^{\circ} 00^{\prime} \mathrm{E}$ and $89^{\circ} 55^{\prime} \mathrm{E}$ (Hussain and Acharya, 1994; Hoq, 2008). The total area of SMFB is about 6,017 sq km that is composed of the landmass of 4,143 sq km (Katebi, 2001) and water bodies of 1,874 sq km in the forms of complex network of rivers, canals and tidal creeks (Iftekhar and Islam, 2004). It consists of about $4.07 \%$ of total land mass of the country and $40 \%$ of total forest land (BFD, 2015).

This study, conducted during 2010-2014, was based on a thorough taxonomic inventory on wild, planted and cultivated vascular plants growing in the SMFB. A total of 3,103 representative specimens were collected during 15 extensive field trips, each consisted of 12-15 days, covering all types of forests and habitats of most of the areas throughout the SMFB (Fig. 1). These specimens were carefully examined in Plant Systematics and Biodiversity Laboratory of Jahangirnagar University and Bangladesh National Herbarium (DACB), Dhaka. The freshly collected specimens were properly processed and managed using standard herbarium techniques (Hyland, 1972; Jain and Raw, 1977) and deposited at Jahangirnagar University Herbarium (JUH). Besides, the first and second authors' own collections and the herbarium specimens previousely collected from the same area by different collectors and deposited at the JUH and the DACB were also examined.

All plant specimens were identified through consulting the experts, and matching with relevant voucher specimens preserved at the JUH and the DACB, taxonomic descriptions and keys available in the relevant literatures (Hooker, 1872-1897; Prain, 1903; Nasir and Ali, 1980-2005; Wu et al., 1995-2013; Siddiqui et al., 2007, 2008; Ahmed et al., 2008-2009; Watson et al., 2011) and clear type images available on the websites of different international herbaria. Nomenclatural information was incorporated following Index Kewensis, recent taxonomic publications (viz., Nasir and Ali, 1980-2005; Flora of North America Editorial Committee, 1993-2014; Wu et al., 1995-2013; Watson et al., 2011) and the nomenclatural databases of IPNI (2008) and TROPICOS (2010). True mangrove and mangrove associate species were recognized following FAO (2007), Giesen et al. (2007) and Barik and Chowdhury (2014). The families of pteridophytes have been 
placed first according to the classification system of Pichi (1977), whereas, the angiosperm families have been arranged following the classification system of Cronquist (1981). The genera and species under each family have been arranged alphabetically. Distribution of the species has been mentioned in three saline zones, i.e. oligohaline $(<5 \mathrm{ppt})$, mesohaline $(5-10 \mathrm{ppt})$ and polyhaline (>10 ppt) zones, as categorized by Karim (1988).

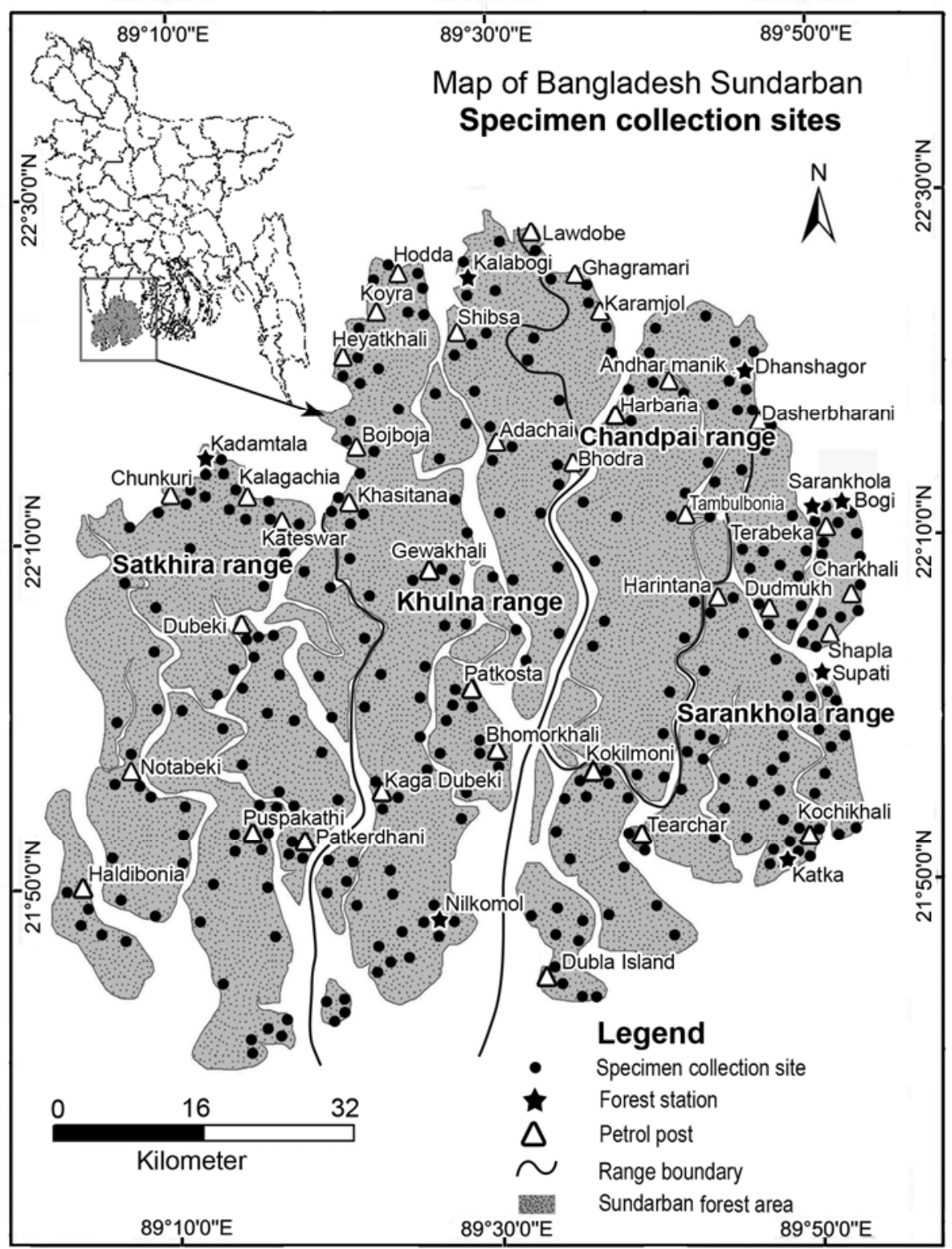

Fig.1. Map of the Sundarban Mangrove Forest of Bangladesh (SMFB) showing specimen collection sites 


\section{Results and Discussion}

The study revealed the occurrence of 528 species belonging to 356 genera under 111 families of vascular plants in the SMFB. Among the species enumerated, 504 (95.46\%) were angiosperms and the rest $24(4.54 \%)$ were pteridophytes (Table 1). Twenty-four species were true mangroves, 70 were mangrove associates and 434 were non-mangrove species (Table 2). Thirty-two species of mangrove associates and all non-mangrove species included in this checklist were previousely reported from the Bangladesh territory (Siddiqui et al., 2007-2008; Ahmed et al., 2008-2009).

Table 1. Taxonomic enumeration of major plant groups of Sundarban Mangrove Forest of Bangladesh.

\begin{tabular}{lccc}
\hline Plant group & Family & Genus & Species \\
\hline Pteridophyta & 14 & 20 & 24 \\
Magnoliopsida (Dicotyledons) & 77 & 260 & 373 \\
Liliopsida (Monocotyledons) & 20 & 76 & 131 \\
\hline
\end{tabular}

In this study, 48 families were found as monogeneric and monospecific and only 15 families were found to be comprised of 10-42 species. Pteridaceae with five species was found as the largest family of Pteridophyta in the SMFB followed by Polypodiaceae with four species and Salviniaceae with three species. Fabaceae with 42 species was recorded as the largest dicotyledonous family in the SMFB followed by Euphorbiaceae with 29 species and Asteraceae with 27 species. In monocotyledons, Poaceae was the largest family with 42 species followed by Cyperaceae with 39 species and Orchidaceae with 9 species. The specimen Sayedur18 (JUH) of Pteridophyta and Sayedur551 (JUH) of Magnoliopsida are recognized here as dubious taxa.

In the SMFB, 345 species $(65.34 \%$ ) were found as herb followed by trees (94 species or $17.80 \%$ ) and shrubs ( 89 species or $16.86 \%$ ). A total of 251 species were found to be distributed exclusively in oligohaline zone, 103 species in mesohaline zone, 57 species in polyhaline zone, and 120 species in more than one saline zone. Sarankhola Forest Range haboured 447 species, Khulna Range 250 species, Chandpai Range 151 species, Satkhira Range 130 species, and 108 species were found in all four forest ranges of the SMFB.

Throughout the SMFB, the forest margins were found as the most common habitat harbouring a total of 255 species, of which 47 were planted and six were cultivated. All of the planted and cultivated species were found to grow only on the uninundated areas and some of these species were found to be naturalized there (e.g. Acacia nilotica (L.) Willd. ex Delile, Pithecellobium dulce (Roxb.) Benth. and Syzygium cumini (L.) Skeels). The genus Colubrina Rich. ex Brongn. and its species C. javanica Miq. of family Rhamnaceae were recently reported as new angiosperm records for Bangladesh (Rahman et al., 2014). Most of the species found in the SMFB are economically important, especially as sources of timber, fuel wood, fruits and vegetables, and also as fodders, medicinal plants, ornamental plants and soil binders. A large number of species were recorded as weeds (Fig. 2).

Eleven species, viz., Asplenium polyodon G. Forst., Dendrobium anceps Sw., Dolichandrone spathacea (L. f.) Seem., Drypetes assamica (Hook. f.) Pax \& K. Hoffm., Indigofera trifoliata L., Luisia brachystachys (Lindl.) Blume, Merope angulata (Willd.) Swingle, Murdannia vaginata (L.) G. Brückn., Pelatantheria insectifera (Rchb. f.) Ridl., Trias oblonga Lindl. and Zeuxine strateumatica (L.) Schltr., recognized as threatened in Bangladesh (Siddiqui et al., 2007, 2008; Ahmed et al., 2008-2009; Ara et al., 2013), were found in the Sundarban. The number of threatened plant species in the SMFB might be much higher in the near future if effective conservation initiatives are not properly implemented. 


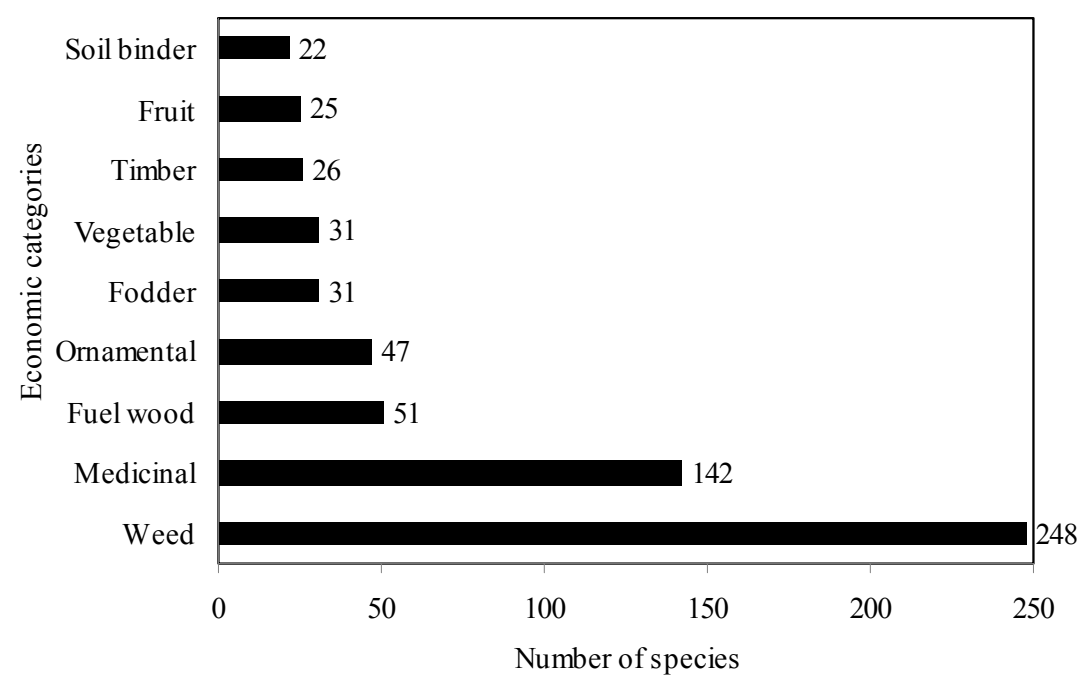

Fig. 2. Economic categories of vascular plant species found in Sundarban Mangrove Forest of Bangladesh (SMFB). Several species had more than one economic uses.

The total number of species recorded in this study for Bangladesh Sundarban (i.e. 528 species) was higher than that (334 species) of Prain (1903) who recorded for both Bangladesh and Indian parts of Sundarban. Out of Prain (1903)'s 334 species, no fewer than 123 species occured in Bangladesh Sundarban according to Karim (1994). The present study has confirmed the occurance of 220 species of Prain (1903)'s 334 species in the SMFB. Among the remaining 114 species, 82 and 15 species were reported to occur in Bangladesh and Indian parts of Sundarban, respectively, five were mentioned as rare, and 12 were suggested to 'look for' in the Sundarban by Prain (1903).

The number of plant species recorded for the SMFB by the previous studies conducted after Prain (1903) was much lower (e.g. 65 and 48 species of true mangroves, mangrove associates and non-mangroves, respectively, by Chaffy et al., 1985 and Rashid et al., 2008) than that of the present study. The consideration of a number of quadrat sampling plots on selected areas (Chaffy et al., 1985; Rashid et al., 2008) or only a particular plant groups (e.g. undergrowths by Rashid et al., 2008) in those studies might be the potential reasons for this notable differences in the findings. In comparison with the species composition of other forests of Bangladesh (e.g. Uddin et al., 1998; Uddin and Hassan, 2004), the total number of species found in SMFB seems to be lower in respect to its area. Impacts of salinity, water logging, tidal action, poor humus, and natural disaster on the SMFB might be the reasons for comparatively lower species richness (Chowdhury, 2001).

The present checklist is an attempt to list down all vascular plant species currently occurring in the SMFB, thus updating our floristic knowledge of this unique ecosystem. It can be considered as an important database as well as baseline to track changes in the floristic composition of the SMFB in course of time, especially in the light of the introduction of new species and/or loss of the current species as a result of different biogeographical processes or risks, especially by multifarious anthropogenic activities and different natural hazards operating there. This checklist may further stimulate research and management in the SMFB by serving as a valuable 'snapshot' of the flora of this globally important mangrove. 
Table 2. List of vascular plants of the Sundarban Mangrove Forest of Bangladesh.

\begin{tabular}{|c|c|c|c|c|c|c|c|}
\hline Scientific name & Bangla name & $\mathrm{MC}$ & Habit & Distribution & Habitat & Use & RS \\
\hline \multicolumn{8}{|l|}{ PTERIDOPHYTA } \\
\hline \multicolumn{8}{|l|}{ Psilotaceae J.W. Griff. \& Henfr. } \\
\hline "Psilotum nudum (L.) P. Beauv. & Psilotum & NMS & Herb, ep & $\mathrm{Mz} ; \mathrm{Sr}$ & $\mathrm{pb}$ & M & Sayedur1242 \\
\hline \multicolumn{8}{|l|}{ Selaginellaceae Willk. } \\
\hline Selaginella vaginata Spring & Selaginella & NMS & Herb, $p$ & $\mathrm{Az} ; \mathrm{Sr}, \mathrm{Kh}$ & $\mathrm{ml}$ & M & Sayedur1223 \\
\hline \multicolumn{8}{|l|}{ Ophioglossaceae Martinov } \\
\hline Ophioglossum reticulatum L. & Sharpa jihba & NMS & Herb, e & $\mathrm{Pz} ; \mathrm{Kh}, \mathrm{St}$ & $\mathrm{gl}, \mathrm{ml}$ & $\mathrm{V}, \mathrm{M}$ & Sayedur603 \\
\hline \multicolumn{8}{|l|}{ Salviniaceae Martinov } \\
\hline Salvinia cucullata Roxb. & Indur kani & NMS & Herb, a & $\mathrm{Oz}, \mathrm{Pz} ; \mathrm{Sr}, \mathrm{St}$ & wt & $\mathrm{Om}$ & Sayedur1198 \\
\hline S. molesta D.S. Mitch. & Pani dhekia & NMS & Herb, a & $\mathrm{Oz} ; \mathrm{Sr}$ & wt & $\mathrm{Om}$ & Sayedur1085 \\
\hline S. natans (L.) All. & Pani dhekia & NMS & Herb, a & $\mathrm{Oz}, \mathrm{Mz} ; \mathrm{Sr}$ & wt & $\mathrm{Om}$ & Mosharof421 \\
\hline \multicolumn{8}{|l|}{ Azollaceae Wettst. } \\
\hline Azolla pinnata R. Br. & Lal khudipana & NMS & Herb, a & $\mathrm{Oz}, \mathrm{Mz} ; \mathrm{Sr}, \mathrm{Ch}$ & wt & $\mathrm{Bf}$ & Mosharof414 \\
\hline \multicolumn{8}{|l|}{ Marsileaceae Mirb. } \\
\hline Marsilea quadrifolia $\mathrm{L}$. & Susni shak & NMS & Herb, cr & $\mathrm{Mz}, \mathrm{Pz} ; \mathrm{Sr}, \mathrm{Kh}$ & wt & V & Mosharof143 \\
\hline \multicolumn{8}{|l|}{ Lygodiaceae M. Roem. } \\
\hline Lygodium flexuosum (L.) Sw. & Dhekia lata & NMS & Herb, c & $\mathrm{Oz}, \mathrm{Mz} ; \mathrm{Ch}, \mathrm{Sr}$ & wd, fm & M & Sayedur187 \\
\hline \multicolumn{8}{|l|}{ Pteridaceae E.D.M. Kirchn. } \\
\hline "Acrostichum aureum L. & Hodo & MS & Herb, e & $\mathrm{Az} ; \mathrm{Ar}$ & wd, fm & $\mathrm{V}, \mathrm{M}$ & I Sayedur1123 \\
\hline Adiantum tenerum $\mathrm{Sw}$. & Biddapata & NMS & Herb, $p$ & $\mathrm{Mz} ; \mathrm{Sr}$ & $\mathrm{ml}$ & $\mathrm{O}$ & Sayedur1197 \\
\hline Ceratopteris pteridoides (Hook.) Hieron. & Pani dhekia & NMS & Herb, a & $\mathrm{Pz} ; \mathrm{St}$ & wt & $\mathrm{V}$ & Sayedur1107 \\
\hline${ }^{*}$ C. thalictroides (L.) Brongn. & Pani dhekia & NMS & Herb, a & $\mathrm{Az} ; \mathrm{Ar}$ & wt & $\mathrm{V}$ & Sayedur396 \\
\hline${ }^{*}$ Pteris vittata $\mathrm{L}$. & Dhekia & NMS & Herb, $p$ & $\mathrm{Oz}, \mathrm{Pz} ; \mathrm{Sr}, \mathrm{Kh}$ & $\mathrm{ml}$ & M & Sayedur261 \\
\hline \multicolumn{8}{|l|}{ Vittariaceae Ching } \\
\hline${ }^{*}$ Haplopteris elongata (Sw.) E.H. Crane & Unknown & MAS & Herb, ep & $\mathrm{Az} ; \mathrm{Ar}$ & $\mathrm{tt}$ & M & Sayedur1256 \\
\hline \multicolumn{8}{|c|}{ Polypodiaceae J. Presl \& C. Presl } \\
\hline${ }^{*}$ Drynaria quercifolia (L.) J. Smith & Pankhiraj & NMS & Herb, ep & $\mathrm{Az} ; \mathrm{Ar}$ & $\mathrm{tt}$ & $\mathrm{O}, \mathrm{M}$ & Sayedur17 \\
\hline "Microsorum punctatum (L.) Copel. & Gucha patra & NMS & Herb, ep & $\mathrm{Az} ; \mathrm{Ar}$ & $\mathrm{tt}$ & $\mathrm{O}, \mathrm{M}$ & Sayedur360 \\
\hline *Pyrrosia nuda (Giesenh.) Ching & Unknown & NMS & Herb, ep & $\mathrm{Az} ; \mathrm{Ar}$ & $\mathrm{tt}$ & M & Sayedur16 \\
\hline \multicolumn{8}{|l|}{ Blechnaceae Newman } \\
\hline "Stenochlaena palustris (Burm.f.) Bed. & Dhekia lata & MAS & Herb, c & $\mathrm{Oz}, \mathrm{Mz} ; \mathrm{Sr}, \mathrm{Ch}$ & wd, fm & $\mathrm{V}$ & Sayedur336 \\
\hline
\end{tabular}

Legend: MC (mangrove classification): MS = true mangrove species, MAS = mangrove associate species, NMS $=$ nonmangrove species; Habit: a $=$ aquatic, $\mathrm{c}=$ climbing, $\mathrm{cr}=$ creeping, $\mathrm{e}=$ erect, ep $=$ epiphytic, $1=$ large, $\mathrm{m}=$ medium, $\mathrm{ph}=$ prostrate, $\mathrm{ps}=$ parasitic, $\mathrm{s}=$ small, $\mathrm{sc}=$ scandent, $\mathrm{wc}=$ woody climber; Distribution: $\mathrm{Ar}=$ all range, $\mathrm{Az}=$ all zones, $\mathrm{Mz}=$ mesohaline zone, $\mathrm{Oz}=$ oligohaline zone, $\mathrm{Pz}=$ polyhaline zone; $\mathrm{Ch}=$ Chandpai Range, $\mathrm{Kh}=$ Khulna Range, $\mathrm{Sr}=$ Sarankhola Range, $\mathrm{St}=$ Satkhira Range; Habitat: $\mathrm{cl}=$ cultivated, $\mathrm{fm}=$ forest margin, $\mathrm{gl}=$ grassland, $\mathrm{ml}=$ marginal land, os $=$ open scrub, $\mathrm{pb}=$ on plant base, $\mathrm{pc}=$ on plant canopy, $\mathrm{pl}=$ planted, $\mathrm{rb}=$ river bank, $\mathrm{sd}=$ sandy dune, $\mathrm{tb}=$ on tree branch, $\mathrm{tt}=$ on tree trunk, $\mathrm{wd}=$ woodland, $\mathrm{wt}=$ wetland; Use: $\mathrm{Aw}=$ aquatic weed, $\mathrm{Bf}=$ bio-fertilizer, $\mathrm{D}=\mathrm{dye}, \mathrm{Du}=$ domestic uses, $\mathrm{F}=$ fibre, $\mathrm{Fd}=$ fodder, $\mathrm{Ff}=$ fish feed, $\mathrm{Fn}=$ furniture, $\mathrm{Fr}=$ fruit, $\mathrm{Fw}=$ fuel wood, $\mathrm{Hp}=$ honey production, $\mathrm{Ju}$ = juice, $\mathrm{M}=$ medicine, $\mathrm{Mn}=$ manure, $\mathrm{Mt}=$ mat, $\mathrm{O}=$ ornamental, $\mathrm{Oi}=$ oil, $\mathrm{Om}=$ organic manure, $\mathrm{P}=$ pulse, $\mathrm{Sb}=$ soil binder, $\mathrm{Sp}=$ spice, $\mathrm{Sw}=$ soft wood, $\mathrm{T}=$ timber, $\mathrm{V}=$ vegetable, $\mathrm{W}=$ weed; $\mathbf{R S}=$ representative specimen; $*$ = previously reported by Prain (1903). 
Table 2. Contd.

\begin{tabular}{|c|c|c|c|c|c|c|c|}
\hline Scientific name & Bangla name & $\mathrm{MC}$ & Habit & Distribution & Habitat & Use & RS \\
\hline \multicolumn{8}{|l|}{ Thelypteridaceae Ching ex Pic. Serm. } \\
\hline $\begin{array}{l}\text { *Ampelopteris prolifera (Retz.) } \\
\text { Copel. }\end{array}$ & Dhekia shak & NMS & Herb, $p$ & $\mathrm{Oz}, \mathrm{Mz} ; \mathrm{Sr}, \mathrm{Ch}$ & $\mathrm{ml}$ & M & Sayedur1326 \\
\hline Cyclosorus crinipes (Hook.) Ching & Bish dhekia & NMS & Herb, e & $\mathrm{Oz}, \mathrm{Mz} ; \mathrm{Sr}, \mathrm{Ch}$ & $\mathrm{ml}$ & $\mathrm{O}$ & Sayedur 1258 \\
\hline C. dentatus (Forssk.) Ching & Bish dhekia & NMS & Herb, e & $\mathrm{Oz}, \mathrm{Mz} ; \mathrm{SrCh}$ & $\mathrm{ml}$ & $\mathrm{O}$ & Sayedur1327 \\
\hline \multicolumn{8}{|l|}{ Aspleniaceae Newman } \\
\hline $\begin{array}{l}\text { *Asplenium polyodon G. Forst. } \\
\text { Athyriaceae Alston }\end{array}$ & Bon dhekia & MAS & Herb, ep & $\mathrm{Oz}, \mathrm{Mz} ; \mathrm{Sr}$ & $\mathrm{tt}$ & $\mathrm{O}, \mathrm{M}$ & Sayedur1037 \\
\hline $\begin{array}{l}{ }^{*} \text { Diplazium esculentum (Retz.) Sw. } \\
\text { MAGNOLIOPSIDA (DICOTYLE }\end{array}$ & $\begin{array}{l}\text { Dhekia shak } \\
\text { DONS) }\end{array}$ & NMS & Herb, e & $\mathrm{Oz} ; \mathrm{Sr}, \mathrm{Ch}$ & $\mathrm{ml}$ & $\mathrm{V}$ & Sayedur963 \\
\hline \multicolumn{8}{|c|}{ Annonaceae Juss. } \\
\hline $\begin{array}{l}\text { Polyalthia longifolia (Sonn.) } \\
\text { Thwaites }\end{array}$ & Debdaru & NMS & Tree, 1 & $\mathrm{Oz} ; \mathrm{Sr}$ & $\mathrm{ml}(\mathrm{pl})$ & $\mathrm{O}$ & Sayedur269 \\
\hline \multicolumn{8}{|l|}{ Lauraceae Juss. } \\
\hline $\begin{array}{l}\text { Litsea glutinosa (Lour.) Robin. } \\
\text { Piperaceae Giseke }\end{array}$ & Menda & NMS & Tree, $\mathrm{s}$ & $\mathrm{Oz} ; \mathrm{Sr}$ & os & M & Sayedur301 \\
\hline $\begin{array}{l}\text { Peperomia pellucida (L.) Kunth } \\
\text { Aristolochiaceae Juss. }\end{array}$ & Luchipata & NMS & Herb, e & $\mathrm{Oz} ; \mathrm{Sr}$ & $\mathrm{ml}$ & M & Sayedur356 \\
\hline $\begin{array}{l}\text { *Aristolochia indica L. } \\
\text { Nymphaeaceae Salisb. }\end{array}$ & Ishwarmul & NMS & Herb, c & $\mathrm{Az} ; \mathrm{Ar}$ & $\mathrm{fm}$ & M & Sayedur486 \\
\hline Nymphaea nouchali Burm. f. & Nilshapla & NMS & Herb, a & $\mathrm{Oz} ; \mathrm{Sr}$ & wt & $\mathrm{M}, \mathrm{O}$ & Sayedur198 \\
\hline N. rubra Roxb. ex Andrews & Lalshapla & NMS & Herb, a & $\mathrm{Az} ; \mathrm{Ar}$ & wt & $\mathrm{M}, \mathrm{O}$ & Sayedur1114 \\
\hline \multicolumn{8}{|l|}{ Ceratophyllaceae Gray } \\
\hline $\begin{array}{l}{ }^{*} \text { Ceratophyllum demersum L. } \\
\text { Ranunculaceae Juss. }\end{array}$ & Kantajhanjhi & NMS & Herb, a & $\mathrm{Oz} ; \mathrm{Sr}$ & wt & Aw & Sayedur348 \\
\hline \multicolumn{8}{|l|}{ Menispermaceae Juss. } \\
\hline Stephania japonica (Thunb.) Miers & Nimukha & NMS & Herb, c & $\mathrm{Oz}, \mathrm{Mz} ; \mathrm{Sr}, \mathrm{Ch}$ & $\mathrm{fm}, \mathrm{os}$ & M & Sayedur411 \\
\hline \multicolumn{7}{|l|}{ Ulmaceae Mirb. } & Sayedur338 \\
\hline${ }^{*}$ Trema orientalis (L.) Blume & Banjiga & NMS & Tree, s & $\mathrm{Az} ; \mathrm{Ar}$ & os, gl & $\mathrm{W}$ & Mosharof 210 \\
\hline \multicolumn{8}{|l|}{ Moraceae Link } \\
\hline Artocarpus heterophyllus Lam. & Kanthal & NMS & Tree, $\mathrm{m}$ & $\mathrm{Oz} ; \mathrm{Sr}, \mathrm{Ch}$ & $\mathrm{ml}(\mathrm{pl})$ & Fr; T & Sayedur1296 \\
\hline Ficus benghalensis L. & Bot/Jhuribot & NMS & Tree, 1 & $\mathrm{Az} ; \mathrm{Ar}$ & $\mathrm{ml}$ & $\mathrm{O}, \mathrm{Fw}$ & Sayedur1137 \\
\hline F. benjamina L. & Bot & NMS & Tree, $\mathrm{m}$ & $\mathrm{Oz} ; \mathrm{Sr}$ & $\mathrm{rb}$ & Fw & Sayedur892 \\
\hline F. elastica Roxb. ex Hornem. & Rubber & NMS & Tree, $\mathrm{m}$ & $\mathrm{Az} ; \mathrm{Ar}$ & $\mathrm{ml}(\mathrm{pl})$ & $\mathrm{O}$ & Sayedur1926 \\
\hline F. heterophylla L. f. & Lotabot & NMS & Herb, c & $\mathrm{Oz} ; \mathrm{Sr}$ & $\mathrm{ml}$ & $\mathrm{W}$ & Sayedur817 \\
\hline F. hispida L. f. & Kakdumur & NMS & Tree, $\mathrm{s}$ & $\mathrm{Oz} ; \mathrm{Sr}, \mathrm{Ch}$ & $\mathrm{fm}, \mathrm{ml}$ & Fr, Fw & Sayedur759 \\
\hline${ }^{*}$ F. microcarpa L.f. & Jir/kamrup & MAS & Shrub & $\mathrm{Mz} ; \mathrm{Kh}$ & $\mathrm{ml}$ & Fw & $N 1176$ \\
\hline F. racemosa $\mathrm{L}$. & Jagdumur & NMS & Tree, $\mathrm{s}$ & $\mathrm{Oz}, \mathrm{Sr}$ & $\mathrm{rb}, \mathrm{fm}$ & $\mathrm{M}, \mathrm{Fw}$ & Sayedur965 \\
\hline${ }^{*}$ F. religiosa $\mathrm{L}$. & Assawath & NMS & Tree, 1 & $\mathrm{Oz} ; \mathrm{Sr}, \mathrm{Ch}$ & $\mathrm{ml}$ & $\mathrm{O}, \mathrm{Fw}$ & Mosharof100 \\
\hline "Streblus asper Lour. & Sheora & NMS & Tree, $\mathrm{m}$ & $\mathrm{Oz}, \mathrm{Mz} ; \mathrm{Sr}, \mathrm{Kh}$ & $\mathrm{fm}, \mathrm{os}$ & Fw & Sayedur695 \\
\hline
\end{tabular}


Table 2. Contd.

\begin{tabular}{|c|c|c|c|c|c|c|c|}
\hline Scientific name & Bangla name & $\mathrm{MC}$ & Habit & Distribution & Habitat & Use & RS \\
\hline \multicolumn{8}{|l|}{ Urticaceae Juss. } \\
\hline Gonostegia sp. & Unknown & NMS & Herb, e & $\mathrm{Oz} ; \mathrm{Sr}$ & $\mathrm{ml}$ & M & Sayedur566 \\
\hline Pilea microphylla (L.) Liebm. & Latamaricha & NMS & Herb, $p$ & $\mathrm{Az} ; \mathrm{Ar}$ & $\mathrm{ml}$ & $\mathrm{W}$ & Mosharof170 \\
\hline $\begin{array}{l}\text { Pouzolzia zeylanica (L.) Benn. \& } \\
\text { R.Br. }\end{array}$ & Kullaruki & NMS & Herb, e & $\mathrm{Az} ; \mathrm{Ar}$ & $\mathrm{ml}$ & $\mathrm{W}$ & Sayedur973 \\
\hline \multicolumn{8}{|l|}{ Casuarinaceae R. Br. } \\
\hline "Casuarina equisetifolia $\mathrm{L}$. & Jhaw & MAS & Tree, 1 & $\mathrm{Mz} ; \mathrm{Sr}$ & $\mathrm{ml}(\mathrm{pl})$ & $\mathrm{O}$ & Sayedur1201 \\
\hline \multicolumn{8}{|l|}{ Nyctaginaceae Juss. } \\
\hline Boerhavia diffusa L. & Punarnava & NMS & Herb, $p$ & $\mathrm{Oz} ; \mathrm{Sr}, \mathrm{Ch}$ & $\mathrm{ml}$ & M & Mosharof30 \\
\hline Bougainvillea spectabilis Willd. & Baganbilash & NMS & Shrub, s & $\mathrm{Az} ; \mathrm{Ar}$ & $\mathrm{ml}(\mathrm{pl})$ & $\mathrm{O}$ & Sayedur1898 \\
\hline Nyctanthes arbor-tristis L. & Shiuli & NMS & Shrub & $\mathrm{Mz} ; \mathrm{Sr}$ & $\mathrm{ml}(\mathrm{pl})$ & $\mathrm{O}$ & Sayedur1158 \\
\hline \multicolumn{8}{|l|}{ Aizoaceae Martinov } \\
\hline *Sesuvium portulacastrum (L.) L. & Sagornunia & MAS & Herb, e & $\mathrm{Az} ; \mathrm{Sr}, \mathrm{Kh}$ & $\mathrm{ml}$ & $\mathrm{W}$ & Sayedur623 \\
\hline \multicolumn{8}{|l|}{ Chenopodiaceae Vent. } \\
\hline Chenopodium album L. & Botuashak & NMS & Herb, e & $\mathrm{Oz} ; \mathrm{Sr}$ & $\mathrm{ml}$ & $\mathrm{M}, \mathrm{V}$ & Sayedur875 \\
\hline \multicolumn{8}{|l|}{ Amaranthaceae Juss. } \\
\hline Achyranthes aspera L. & Apang & NMS & Herb, e & $\mathrm{Oz} ; \mathrm{Sr}$ & $\mathrm{ml}$ & M & Sayedur521 \\
\hline $\begin{array}{l}\text { Alternanthera paronychioides A. St.- } \\
\text { Hil. }\end{array}$ & Jhuli khata & NMS & Herb, a & $\mathrm{Oz} ; \mathrm{Sr}, \mathrm{Ch}$ & wt & $\mathrm{W}$ & Sayedur1310 \\
\hline A. philoxeroides (Mart.) Griseb. & Helencha & NMS & Herb, a & $\mathrm{Az} ; \mathrm{Ar}$ & wt & V & Sayedur1257 \\
\hline "A. sessilis (L.) R. Br. ex DC. & Sachi shak & NMS & Herb, a & $\mathrm{Oz} ; \mathrm{Sr}, \mathrm{Ch}$ & wt & $\mathrm{V}, \mathrm{W}$ & Sayedur383 \\
\hline Amaranthus spinosus L. & Katanoty & NMS & Herb, e & $\mathrm{Oz}, \mathrm{Mz} ; \mathrm{Sr}, \mathrm{Ch}$ & $\mathrm{ml}$ & $\mathrm{V}, \mathrm{W}$ & Mosharof16 \\
\hline A. tricolor L. & Lalshak & NMS & Herb, e & $\mathrm{Az} ; \mathrm{Ar}$ & $\mathrm{ml}(\mathrm{cl})$ & $\mathrm{V}$ & Sayedur1103 \\
\hline *A. viridis $\mathrm{L}$. & Notey shak & NMS & Herb, e & $\mathrm{Oz}, \mathrm{Mz} ; \mathrm{Sr}, \mathrm{Kh}$ & $\mathrm{ml}$ & $\mathrm{V}$ & Sayedur2226 \\
\hline Celosia argentea $\mathrm{L}$. & Morogful & NMS & Herb, e & $\mathrm{Oz} ; \mathrm{Sr}$ & $\mathrm{ml}$ & $\mathrm{W}$ & Mosharof33 \\
\hline \multicolumn{8}{|l|}{ Portulacaceae Juss. } \\
\hline *Portulaca oleracea L. & Nunia shak & NMS & Herb, $p$ & $\mathrm{Az} ; \mathrm{Ar}$ & $\mathrm{ml}$ & $\mathrm{O}$ & Sayedur65 \\
\hline \multicolumn{8}{|l|}{ Basellaceae Raf. } \\
\hline *Basella alba L. & Puishak & NMS & Herb, cr & $\mathrm{Az} ; \mathrm{Ar}$ & $\mathrm{ml}(\mathrm{cl})$ & $\mathrm{V}$ & Sayedur1330 \\
\hline \multicolumn{8}{|l|}{ Polygonaceae Juss. } \\
\hline Persicaria barbatum (L.) Hara & Bishkathali & NMS & Herb, e & $\mathrm{Oz}, \mathrm{Mz} ; \mathrm{Sr}, \mathrm{Kh}$ & $\mathrm{fm}, \mathrm{ml}$ & W & Sayedur779 \\
\hline P. glabra (Willd.) Gomez de la Maza & Lal kukri & NMS & Herb, e & $\mathrm{Oz}, \mathrm{Pz} ; \mathrm{Sr}, \mathrm{St}$ & $\mathrm{ml}$ & $\mathrm{M}, \mathrm{W}$ & N1277 \\
\hline P. hydropiper (L.) Spach & Bishkathali & NMS & Herb, e & $\mathrm{Oz}, \mathrm{Mz} ; \mathrm{Sr}, \mathrm{Kh}$ & wt, ml & $\mathrm{M}, \mathrm{W}$ & Sayedur1064 \\
\hline P. serrulata (Lag.) Webb \& Moq. & Polygonum & NMS & Herb, e & $\mathrm{Oz}, \mathrm{Mz} ; \mathrm{Sr}$ & $\mathrm{ml}$ & $\mathrm{W}$ & Sayedur492 \\
\hline P. tomentosa (Schrank) E.P. Bicknell & Bishkathali & NMS & Herb, e & $\mathrm{Oz} ; \mathrm{Sr}$ & $\mathrm{ml}$ & $\mathrm{W}$ & Sayedur493 \\
\hline Polygonum effusum Meisn & Chemtishak & NMS & Herb, e & $\mathrm{Oz}, \mathrm{Mz} ; \mathrm{Sr}, \mathrm{Kh}$ & $\mathrm{ml}$ & W & Sayedur2249 \\
\hline Rumex maritimus L. & Gang palong & NMS & Herb, e & $\mathrm{Oz}, \mathrm{Mz} ; \mathrm{Sr}$ & wt, ml & $\mathrm{M}, \mathrm{W}$ & Sayedur788 \\
\hline \multicolumn{8}{|l|}{ Plumbaginaceae Juss. } \\
\hline "Aegialitis rotundifolia Roxb. & Nunia & MS & Shrub & $\mathrm{Pz} ; \mathrm{St}$ & wd, fm & $\mathrm{Fw}$ & Sayedur409 \\
\hline
\end{tabular}


Table 2. Contd.

\begin{tabular}{|c|c|c|c|c|c|c|c|}
\hline Scientific name & Bangla name & $\mathrm{MC}$ & Habit & Distribution & Habitat & Use & RS \\
\hline \multicolumn{8}{|l|}{ Clusiaceae Lindl. } \\
\hline Calophyllum inophyllum L. & Punnal & MAS & Tree, $\mathrm{m}$ & $\mathrm{Az} ; \mathrm{Ar}$ & $\mathrm{fm}, \mathrm{ml}$ & $\mathrm{O}, \mathrm{W}$ & Sayedur1115 \\
\hline \multicolumn{8}{|l|}{ Tiliaceae Juss. } \\
\hline${ }^{*}$ Brownlowia tersa (L.) Kosterm. & Latasundri & MS & Shrub & $\mathrm{Az} ; \mathrm{Ar}$ & $\mathrm{rb}, \mathrm{wd}$ & $\mathrm{F}, \mathrm{Fw}$ & Sayedur364 \\
\hline "Corchorus aestuans L. & Titpata & NMS & Shrub & $\mathrm{Pz} ; \mathrm{Kh}$ & $\mathrm{ml}$ & $\mathrm{W}$ & Sayedur1062 \\
\hline Triumfetta rhomboidea Jacq. & Banokra & NMS & Shrub & $\mathrm{Oz} ; \mathrm{Sr}$ & $\mathrm{ml}$ & $\mathrm{W}$ & Sayedur540 \\
\hline \multicolumn{8}{|l|}{ Sterculiaceae Vent. } \\
\hline *Heritiera fomes Buch.-Ham. & Sundari & MS & Tree, 1 & $\mathrm{Az} ; \mathrm{Ar}$ & wd & $\mathrm{T}, \mathrm{M}$ & Sayedur13 \\
\hline Pterygota alata (Roxb.) R. Br. & Buddhanarikel & NMS & Tree, 1 & $\mathrm{Mz} ; \mathrm{Sr}$ & $\mathrm{ml}(\mathrm{pl})$ & $\mathrm{T}$ & Sayedur656 \\
\hline Sterculia foetida $\mathrm{L}$. & Baxobadam & NMS & Tree, 1 & $\mathrm{Oz} ; \mathrm{Sr}$ & $\mathrm{ml}(\mathrm{pl})$ & $\mathrm{T}, \mathrm{F}$ & Sayedur874 \\
\hline \multicolumn{8}{|l|}{ Bombacaceae Kunth } \\
\hline Bombax ceiba L. & Shimul & NMS & Tree, 1 & $\mathrm{Oz}, \mathrm{Mz} ; \mathrm{Sr}$ & $\mathrm{ml}, \mathrm{fm}$ & $\mathrm{F}, \mathrm{M}$ & Sayedur870 \\
\hline Ceiba pentandra (L.) Gaertn. & Burma shimul & NMS & Tree, $\mathrm{m}$ & $\mathrm{Oz} ; \mathrm{Sr}$ & $\mathrm{ml}(\mathrm{pl})$ & $\mathrm{F}, \mathrm{Fw}$ & Sayedur1309 \\
\hline \multicolumn{8}{|l|}{ Malvaceae Juss. } \\
\hline Abelmoschus moschatus Medik. & Mushak Dana & NMS & Shrub & $\mathrm{Pz} ; \mathrm{Kh}$ & $\mathrm{ml}$ & M & Sayedur81 \\
\hline *Abutilon indicum (L.) Sweet & Gol-peari & NMS & Shrub & $\mathrm{Oz} ; \mathrm{Sr}$ & $\mathrm{ml}$ & $\mathrm{W}$ & Sayedur260 \\
\hline Gossypium arboreum L. & Karpash & NMS & Shrub & $\mathrm{Pz} ; \mathrm{St}$ & $\mathrm{ml}(\mathrm{pl})$ & $\mathrm{F}, \mathrm{M}$ & Sayedur859 \\
\hline${ }^{*}$ Hibiscus tiliaceus L. & Bhola & MAS & Shrub, s & $\mathrm{Az} ; \mathrm{Ar}$ & $\mathrm{rb}, \mathrm{wd}$ & $\mathrm{Fw}, \mathrm{M}$ & Sayedur459 \\
\hline Sida acuta Burm. f. & Kureta & NMS & Shrub & $\mathrm{Oz}, \mathrm{Mz} ; \mathrm{Sr}, \mathrm{Kh}$ & $\mathrm{ml}$, os & $\mathrm{W}$ & Mosharof191 \\
\hline S. cordata (Burm. f.) Borss. Waalk. & Pitberela & NMS & Herb, e & $\mathrm{Az} ; \mathrm{Ar}$ & $\mathrm{ml}$, os & $\mathrm{W}$ & Sayedur1159 \\
\hline S. cordifolia L. & Jhunka & NMS & Herb, e & $\mathrm{Oz} ; \mathrm{Sr}$ & $\mathrm{ml}$, os & $\mathrm{W}$ & Sayedur641 \\
\hline S. rhombifolia $\mathrm{L}$. & Berela & NMS & Herb, e & $\mathrm{Oz}, \mathrm{Mz} ; \mathrm{Sr}, \mathrm{Ch}$ & $\mathrm{ml}, \mathrm{gl}$ & W & Sayedur136 \\
\hline $\begin{array}{l}\text { *Thespesia populnea (L.) Sol. ex } \\
\text { Corrêa }\end{array}$ & Pareshpipul & MAS & Shrub & $\mathrm{Pz} ; \mathrm{St}$ & $\mathrm{fm}$ & $\mathrm{M}, \mathrm{Fw}$ & Sayedur1117 \\
\hline Urena lobata L. & Banokhra & NMS & Shrub & $\mathrm{Oz} ; \mathrm{Sr}, \mathrm{Ch}$ & $\mathrm{ml}$, os & $\mathrm{W}$ & Sayedur515 \\
\hline \multicolumn{8}{|l|}{ Lecythidaceae A. Rich. } \\
\hline${ }^{*}$ Barringtonia racemosa (L.) Spreng. & Hijol & MAS & Tree, $\mathrm{m}$ & $\mathrm{Oz} ; \mathrm{Sr}$ & $\mathrm{rb}, \mathrm{wd}$ & $\mathrm{M}, \mathrm{Fw}$ & Sayedur226 \\
\hline \multicolumn{8}{|l|}{ Flacourtiaceae Rich. ex DC. } \\
\hline${ }^{*}$ Flacourtia indica (Burm. f.) Merr. & Kataboichi & NMS & Shrub & $\mathrm{Oz}, \mathrm{Mz} ; \mathrm{Sr}, \mathrm{Kh}$ & os, $\mathrm{ml}$ & $\mathrm{W}, \mathrm{Fw}$ & Sayedur654 \\
\hline \multicolumn{8}{|l|}{ Tamaricaceae Link } \\
\hline *Tamarix indica Willd. & Nona jhaw & NMS & Tree, $\mathrm{s}$ & $\mathrm{Az} ; \mathrm{Ar}$ & wd, fm & $\mathrm{M}, \mathrm{Fw}$ & Sayedur1475 \\
\hline \multicolumn{8}{|l|}{ Passifloraceae Juss. ex Roussel } \\
\hline Passiflora foetida $\mathrm{L}$. & Jhumkalata & MAS & Herb, c & $\mathrm{Oz} ; \mathrm{Sr}$ & os, fm & $\mathrm{W}$ & Sayedur619 \\
\hline \multicolumn{8}{|l|}{ Caricaceae Dumort. } \\
\hline Carica papaya L. & Papaya & NMS & Shrub & $\mathrm{Az} ; \mathrm{Ar}$ & $\mathrm{ml}(\mathrm{pl})$ & Fr, V & Sayedur367 \\
\hline \multicolumn{8}{|l|}{ Cucurbitaceae Juss. } \\
\hline${ }^{*}$ Coccinia grandis (L.) Voigt & Telakucha & NMS & Herb, c & $\mathrm{Oz} ; \mathrm{Sr}$ & $\mathrm{ml}, \mathrm{fm}$ & $\mathrm{M}, \mathrm{W}$ & Sayedur519 \\
\hline${ }^{*}$ Luffa cylindrica (L.) M. Roem. & Jhinga & NMS & Herb, c & $\mathrm{Oz} ; \mathrm{Sr}$ & $\mathrm{ml}(\mathrm{cl})$ & $\mathrm{V}$ & Sayedur639 \\
\hline${ }^{*}$ Momordica charantia L. & Korola & NMS & Herb, c & $\mathrm{Az} ; \mathrm{Ar}$ & $\mathrm{ml}(\mathrm{cl})$ & $\mathrm{V}, \mathrm{M}$ & Sayedur1089 \\
\hline
\end{tabular}


Table 2. Contd.

\begin{tabular}{|c|c|c|c|c|c|c|c|}
\hline Scientific name & Bangla name & $\mathrm{MC}$ & Habit & Distribution & Habitat & Use & RS \\
\hline \multicolumn{8}{|l|}{ Capparaceae Juss. } \\
\hline${ }^{*}$ Cleome viscosa $\mathrm{L}$. & Hurhuria & NMS & Herb, e & $\mathrm{Oz} ; \mathrm{Sr}$ & $\mathrm{ml}, \mathrm{gl}$ & $\mathrm{W}$ & Sayedur490 \\
\hline *Crateva magna (Lour.) DC. & Borun/Banny & NMS & Tree, $\mathrm{s}$ & $\mathrm{Oz}, \mathrm{Mz} ; \mathrm{Sr}$ & $\mathrm{fm}$ & Fw, M & Sayedur1402 \\
\hline \multicolumn{8}{|l|}{ Brassicaceae Burnett } \\
\hline Rorippa indica (L.) Hiem. & Bansarisha & NMS & Herb, e & $\mathrm{Oz} ; \mathrm{Sr}$ & $\mathrm{ml}$, os & $\mathrm{W}$ & Mosharof181 \\
\hline \multicolumn{8}{|l|}{ Moringaceae Martinov } \\
\hline Moringa oleifera Lam. & Shajna & NMS & Tree, $\mathrm{m}$ & $\mathrm{Oz}, \mathrm{Mz} ; \mathrm{Sr}$ & $\mathrm{ml}(\mathrm{pl})$ & $\mathrm{V}, \mathrm{M}$ & Sayedur532 \\
\hline \multicolumn{8}{|l|}{ Sapotaceae Juss. } \\
\hline $\begin{array}{l}\text { Madhuca longifolia (J. Koenig ex L.) } \\
\text { J.F. Macbr. }\end{array}$ & Mohua & NMS & Tree, $\mathrm{m}$ & $\mathrm{Az} ; \mathrm{Ar}$ & $\mathrm{ml}(\mathrm{pl})$ & $\mathrm{O}$ & Sayedur1111 \\
\hline Manilkara zapota (L.) P. Royen & Sofeda & NMS & Tree, s & $\mathrm{Mz}, \mathrm{Sa}$ & $\mathrm{ml}(\mathrm{pl})$ & Fr & Sayedur740 \\
\hline \multicolumn{8}{|l|}{ Ebenaceae Gürke } \\
\hline Diospyros blancoi A. DC. & Bilatigab & NMS & Tree, $\mathrm{m}$ & $\mathrm{Pz} ; \mathrm{St}$ & $\mathrm{ml}(\mathrm{pl})$ & $\mathrm{Fr}$ & Sayedur2243 \\
\hline${ }^{*}$ D. peregrina Gürke. & $\mathrm{Gab}$ & MAS & Tree, $\mathrm{m}$ & $\mathrm{Oz}, \mathrm{Mz} ; \mathrm{Sr}, \mathrm{Kh}$ & $\mathrm{wd}, \mathrm{rb}$ & $\mathrm{M}, \mathrm{Fr}$ & Sayedur890 \\
\hline \multicolumn{8}{|l|}{ Myrsinaceae R. Br. } \\
\hline "Aegiceras corniculatum (L.) Blanco & Kholshi & MS & Shrub & $\mathrm{Mz}, \mathrm{Pz} ; \mathrm{Kh}, \mathrm{St}$ & $\mathrm{wd}, \mathrm{rb}$ & $\mathrm{Hp}, \mathrm{Fw}$ & Sayedur884 \\
\hline Ardisia solanacea Roxb. & Banjam & MAS & Shrub & $\mathrm{Oz} ; \mathrm{Sr}$ & wd, rb & $\mathrm{M}, \mathrm{Fw}$ & Sayedur968 \\
\hline \multicolumn{8}{|l|}{ Crassulaceae J. St.-Hil. } \\
\hline Kalanchoe pinnata (Lam.) Pers. & Pathorkuchi & NMS & Herb, e & $\mathrm{Oz} ; \mathrm{Sr}$ & $\mathrm{ml}(\mathrm{pl})$ & M & Sayedur361 \\
\hline \multicolumn{8}{|l|}{ Mimosaceae R. Br. } \\
\hline $\begin{array}{l}\text { Acacia auriculiformis A. Cunn. ex } \\
\text { Benth. }\end{array}$ & Akashmoni & NMS & Tree, 1 & $\mathrm{Pz} ; \mathrm{St}$ & $\mathrm{ml}(\mathrm{pl})$ & $\mathrm{T}$ & Sayedur1448 \\
\hline A. catechu (L. f.) Willd. & Khoir & NMS & Tree, $\mathrm{m}$ & $\mathrm{Mz} ; \mathrm{Sr}$ & $\mathrm{ml}(\mathrm{pl})$ & $\mathrm{M}, \mathrm{Fw}$ & Sayedur531 \\
\hline *A. nilotica (L.) Willd. ex Delile & Babla & NMS & Tree, $\mathrm{m}$ & $\mathrm{Az} ; \mathrm{Ar}$ & $\mathrm{ml}(\mathrm{pl})$ & $\mathrm{T}, \mathrm{Fw}$ & Sayedur114 \\
\hline $\begin{array}{l}\text { Albizia niopoides (Spruce ex Benth.) } \\
\text { Burkart }\end{array}$ & Raj shirish & NMS & Tree, 1 & $\mathrm{Oz} ; \mathrm{Sr}$ & wd (pl) & $\mathrm{T}$ & Sayedur1134 \\
\hline A. procera (Roxb.) Benth. & Koroi & NMS & Tree, 1 & $\mathrm{Oz} ; \mathrm{Sr}$ & wd (pl) & $\mathrm{T}$ & Sayedur301 \\
\hline${ }^{*}$ Entada phaseoloides (L.) Merr. & Gila lata & MAS & $\begin{array}{l}\text { Shrub, } \\
\text { wc }\end{array}$ & $\mathrm{Oz}, \mathrm{Mz} ; \mathrm{Sr}, \mathrm{Ch}$ & $\mathrm{wd}, \mathrm{fm}$ & M & Sayedur299 \\
\hline $\begin{array}{l}\text { Leucaena leucocephala (Lam.) de } \\
\text { Wit. }\end{array}$ & Ipil ipil & NMS & Tree, $\mathrm{m}$ & $\mathrm{Pz} ; \mathrm{Kh}$ & $\mathrm{ml}$ & $\mathrm{T}, \mathrm{Fw}$ & Mosharof131 \\
\hline Mimosa pudica L. & Lazzaboti & NMS & Shrub & $\mathrm{Az} ; \mathrm{Ar}$ & $\mathrm{gl}, \mathrm{ml}$ & $\mathrm{W}, \mathrm{M}$ & Sayedur530 \\
\hline Pithecellobium dulce (Roxb.) Benth. & Khoia babla & NMS & Tree, $\mathrm{m}$ & $\mathrm{Pz} ; \mathrm{St}$ & $\mathrm{ml}(\mathrm{pl})$ & Fw, Fr & Sayedur 846 \\
\hline Samanea saman (Jacq.) Merr. & Raintree & NMS & Tree, 1 & $\mathrm{Oz} ; \mathrm{Sr}$ & wd, ml & $\mathrm{T}$ & Mosharof187 \\
\hline \multicolumn{8}{|l|}{ Caesalpiniaceae R. Br. } \\
\hline "Caesalpinia bonduc (L.) Roxb. & Nata & MAS & Shrub, sc & $\mathrm{Mz} ; \mathrm{Sr}$ & $\mathrm{ml}$, os & M & Sayedur513 \\
\hline${ }^{*}$ C. crista $\mathrm{L}$. & Kutumkanta & MAS & Shrub, sc & $\mathrm{Mz}, \mathrm{Pz} ; \mathrm{Sr}$ & $\mathrm{rb}, \mathrm{ml}$ & M & Sayedur1135 \\
\hline${ }^{*}$ Cassia fistula $\mathrm{L}$. & Sonalu & NMS & Tree, $\mathrm{s}$ & $\mathrm{Oz}, \mathrm{Mz} ; \mathrm{Sr}$ & os, $\mathrm{ml}$ & $\mathrm{O}, \mathrm{M}$ & Sayedur1019 \\
\hline${ }^{*}$ Cynometra ramiflora $\mathrm{L}$. & Shingra & MS & Shrub & $\mathrm{Oz}, \mathrm{Mz} ; \mathrm{Sr}, \mathrm{Kh}$ & wdl & Fw & Sayedur1211 \\
\hline Delonix regia (Bojer ex Hook.) Raf. & Krishnachura & NMS & Tree, $\mathrm{m}$ & $\mathrm{Oz}, \mathrm{Mz} ; \mathrm{Sr}$ & $\mathrm{ml}(\mathrm{pl})$ & $\mathrm{O}$ & Sayedur1241 \\
\hline${ }^{*}$ Intsia bijuga (Colebr.) Kuntze. & Bhaila & MAS & Tree, $\mathrm{s}$ & $\mathrm{Oz}, \mathrm{Mz} ; \mathrm{Sr}, \mathrm{Kh}$ & $\mathrm{wd}, \mathrm{rb}$ & $\mathrm{M}, \mathrm{Fw}$ & Sayedur365 \\
\hline *Senna occidentalis L. & Barakolkasuna & NMS & Shrub & $\mathrm{Oz} ; \mathrm{Sr}$ & $\mathrm{ml}$ & $\mathrm{W}, \mathrm{M}$ & Mosharof40 \\
\hline
\end{tabular}


Table 2. Contd.

\begin{tabular}{|c|c|c|c|c|c|c|c|}
\hline Scientific name & Bangla name & $\mathrm{MC}$ & Habit & Distribution & Habitat & Use & RS \\
\hline $\begin{array}{l}\text { S. siamea (Lam.) H.S. Irwin \& } \\
\text { Barneby }\end{array}$ & Minjiri & NMS & Tree, $\mathrm{m}$ & $\mathrm{Oz} ; \mathrm{Sr}$ & $\mathrm{ml}$ & Fw, T & Sayedur328 \\
\hline${ }^{*}$ S. tora $(\mathrm{L}$.$) Roxb.$ & Chakunda & NMS & Herb, e & $\mathrm{Oz}, \mathrm{Mz} ; \mathrm{Sr}$ & $\mathrm{ml}$, os & W & Mosharof190 \\
\hline${ }^{*}$ Tamarindus indica $\mathrm{L}$. & Tetul & NMS & Tree, $\mathrm{m}$ & $\mathrm{Az} ; \mathrm{Ar}$ & $\mathrm{ml}(\mathrm{pl})$ & $\mathrm{Fr}, \mathrm{FW}$ & Sayedur964 \\
\hline \multicolumn{8}{|l|}{ Fabaceae Lindl. } \\
\hline "Abrus precatorius L. & Kuch/rati & MAS & Herb, c & $\mathrm{Oz} ; \mathrm{Sr}$ & $\mathrm{fm}$, os & M & Sayedur1233 \\
\hline Aeschynomene indica L. & Kathshola & NMS & Shrub & $\mathrm{Oz} ; \mathrm{Sr}$ & $\mathrm{ml}$ & $\mathrm{W}, \mathrm{Fw}$ & Sayedur714 \\
\hline $\begin{array}{l}\text { *Aganope heptaphylla (L.) } \\
\text { Polhill }\end{array}$ & Pan-nata & MAS & Shrub, wc & $\mathrm{Mz} ; \mathrm{Sr}, \mathrm{Kh}$ & $\mathrm{rb}$, os & $\mathrm{W}$ & Sayedur520 \\
\hline Alysicarpus sp. & Unknown & NMS & Herb, $p$ & $\mathrm{Pz} ; \mathrm{Kh}$ & $\mathrm{ml}$ & W & Sayedur1156 \\
\hline $\begin{array}{l}{ }^{*} \text { Cajanus scarabaeoides (L.) } \\
\text { Thouars }\end{array}$ & Kukshim & NMS & Herb, c & $\mathrm{Oz} ; \mathrm{Sr}$ & $\mathrm{ml}$, os & W & Sayedur626 \\
\hline${ }^{*}$ Canavalia cathartica Thouars & Kalosim & MAS & Herb, c & $\mathrm{Oz}, \mathrm{Pz} ; \mathrm{Sa}$ & $\mathrm{rb}$ & $\mathrm{F}$ & Sayedur 1270 \\
\hline${ }^{*}$ C. maritima Thouars & Banshim & MAS & Herb, c & $\mathrm{Mz} ; \mathrm{Sr}$ & $\mathrm{sd}, \mathrm{rb}$ & W & Sayedur522 \\
\hline Crotalaria mysorensis Roth & Misori jhonjhoni & NMS & Shrub & $\mathrm{Pz} ; \mathrm{Kh}$ & $\mathrm{ml}, \mathrm{gl}$ & W & N4690 \\
\hline${ }^{*}$ C. pallida Aiton & Jhonjhoni & NMS & Shrub & $\mathrm{Oz}, \mathrm{Pz} ; \mathrm{Sr}, \mathrm{Kh}$ & $\mathrm{ml}, \mathrm{gl}$ & W & Sayedur1163 \\
\hline${ }^{*}$ C. retusa $\mathrm{L}$. & Bil jhonjhoni & NMS & Shrub & $\mathrm{Pz} ; \mathrm{Kh}$ & $\mathrm{ml}, \mathrm{gl}$ & W & Sayedur473 \\
\hline${ }^{*}$ C. verrucosa $\mathrm{L}$. & Boro jhonjhoni & NMS & Shrub & $\mathrm{Oz}, \mathrm{Pz} ; \mathrm{Sr}, \mathrm{Kh}$ & $\mathrm{ml}, \mathrm{gl}$ & $\mathrm{W}$ & Sayedur674 \\
\hline $\begin{array}{l}\text { *Dalbergia candenatensis } \\
\text { (Dennst.) Prain }\end{array}$ & Chanda lata & MAS & Shrub,wc & $\mathrm{Az} ; \mathrm{Ar}$ & $\mathrm{rb}, \mathrm{fm}$ & $\mathrm{W}$ & Sayedur61 \\
\hline${ }^{*} D$. spinosa Roxb. & Kutumkanta & NMS & Shrub,wc & $\mathrm{Oz}, \mathrm{Mz} ; \mathrm{Sr}, \mathrm{Kh}$ & $\mathrm{rb}, \mathrm{fm}$ & W & Sayedur598 \\
\hline $\begin{array}{l}{ }^{*} \text { Derris scandens (Roxb.) } \\
\text { Benth. }\end{array}$ & Mahajoni lata & MAS & Shrub,wc & $\mathrm{Az} ; \mathrm{Ar}$ & $\mathrm{wd}, \mathrm{fm}$ & $\mathrm{F}, \mathrm{W}$ & Sayedur54 \\
\hline${ }^{*} D$. trifoliata Lour. & Kalia lata & MAS & Herb, c & $\mathrm{Az} ; \mathrm{Ar}$ & $\mathrm{wd}, \mathrm{fm}$ & $\mathrm{W}, \mathrm{M}$ & Sayedur931 \\
\hline $\begin{array}{l}\text { Desmodium gangeticum (L.) } \\
\text { DC. }\end{array}$ & Salpani & NMS & Shrub & $\mathrm{Oz} ; \mathrm{Sr}$ & $\mathrm{ml}, \mathrm{gl}$ & W & Sayedur582 \\
\hline D. heterophyllum (Willd.) DC. & Bon motorshuti & NMS & Herb, $p$ & $\mathrm{Pz} ; \mathrm{Kh}$ & os, $\mathrm{ml}$ & $\mathrm{W}$ & Sayedur121 \\
\hline D. microphyllum (Thunb.) DC. & Chotomodi & NMS & Herb, $p$ & $\mathrm{Pz} ; \mathrm{Kh}$ & $\mathrm{os}, \mathrm{ml}$ & W & N4683 \\
\hline D. triflorum (L.) DC. & Kodaliya & NMS & Herb, $p$ & $\mathrm{Az} ; \mathrm{Ar}$ & $\mathrm{gl}, \mathrm{ml}$ & W & Sayedur664 \\
\hline Erythrina fusca Lour. & Patiymandar & NMS & Tree, $\mathrm{s}$ & $\mathrm{Oz} ; \mathrm{Sr}$ & $\mathrm{rb}, \mathrm{ml}$ & $\mathrm{O}, \mathrm{Fw}$ & Sayedur879 \\
\hline${ }^{*}$ E. variegata $\mathrm{L}$. & Jalmandar & MAS & Tree, $\mathrm{s}$ & $\mathrm{Oz} ; \mathrm{Sr}$ & $\mathrm{rb}, \mathrm{ml}$ & $\mathrm{O}, \mathrm{Fw}$ & Sayedur2189 \\
\hline Indigofera trifoliata $\mathrm{L}$. & Ban nil & NMS & Shrub & $\mathrm{Oz}, \mathrm{Pz} ; \mathrm{Sr}, \mathrm{Kh}$ & $\mathrm{ml}$, os & W & N1207 \\
\hline Lablab purpureus (L.) Sweet & Shim & NMS & Herb, c & $\mathrm{Az} ; \mathrm{Ar}$ & $\mathrm{ml}(\mathrm{pl})$ & $\mathrm{V}$ & N1368 \\
\hline Lathyrus sativus L. & Kheshari & NMS & Herb, c & $\mathrm{Oz} ; \mathrm{Sr}$ & $\mathrm{ml}$ & $\mathrm{P}$ & Sayedur770 \\
\hline Medicago polymorpha L. & Treful & NMS & Herb, e & $\mathrm{Mz} ; \mathrm{Sr}$ & $\mathrm{ml}$, os & W & N4887 \\
\hline Melilotus albus Medik. & Sada methi & NMS & Herb, e & $\mathrm{Oz} ; \mathrm{Ch}$ & $\mathrm{ml}, \mathrm{os}$ & W & Sayedur1292 \\
\hline $\begin{array}{l}{ }^{*} \text { Mucuna gigantea (Willd.) } \\
\text { DC. }\end{array}$ & Bara alkushi & NMS & Herb,c & $\mathrm{Oz}, \mathrm{Mz} ; \mathrm{Sr}, \mathrm{Ch}$ & $\mathrm{wd}, \mathrm{rd}$ & $\mathrm{M}, \mathrm{W}$ & Sayedur374 \\
\hline M. pruriens (L.) DC. & Alkushi & NMS & Herb, c & $\mathrm{Pz} ; \mathrm{Kh}$ & $\mathrm{os}, \mathrm{ml}$ & $\mathrm{M}, \mathrm{W}$ & Sayedur89 \\
\hline Phaseolus coccineus L. & Begunilat shim & NMS & Herb, c & $\mathrm{Oz} ; \mathrm{Sr}$ & $\mathrm{ml}$ & $\mathrm{P}$ & Sayedur747 \\
\hline${ }^{*}$ Pongamia pinnata (L.) Pierre & Koromja & MAS & Tree, $\mathrm{m}$ & $\mathrm{Oz}, \mathrm{Mz} ; \mathrm{Sr}, \mathrm{Ch}$ & $\mathrm{wd}, \mathrm{ml}$ & $\mathrm{M}, \mathrm{Fw}$ & Sayedur620 \\
\hline
\end{tabular}


Table 2. Contd.

\begin{tabular}{|c|c|c|c|c|c|c|c|}
\hline Scientific name & Bangla name & $\mathrm{MC}$ & Habit & Distribution & Habitat & Use & $\mathrm{RS}$ \\
\hline Rhynchosia minima (L.) DC. & Minibhatraj & NMS & Herb, c & $\mathrm{Mz} ; \mathrm{Sr}$ & $\mathrm{ml}$, os & W & N4685 \\
\hline R. rufescens (Willd.) DC. & Shimbhatraj & NMS & Herb, c & $\mathrm{Mz} ; \mathrm{Sr}$ & $\mathrm{ml}$, os & $\mathrm{W}$ & N4689 \\
\hline $\begin{array}{l}\text { Tadehagi pseudotriquetrum } \\
\text { (DC.) H. Ohashi }\end{array}$ & Ram manda & NMS & Shrub & $\mathrm{Pz} ; \mathrm{Kh}$ & os, $\mathrm{ml}$ & $\mathrm{W}, \mathrm{M}$ & Sayedur1005 \\
\hline T. triquetrum (L.) H. Ohashi & Luri manda & NMS & Shrub & $\mathrm{Pz} ; \mathrm{Kh}$ & os, $\mathrm{ml}$ & $\mathrm{W}, \mathrm{M}$ & Sayedur1004 \\
\hline Vicia hirsuta (L.) Gray & Masurchana & NMS & Herb, c & $\mathrm{Oz} ; \mathrm{Sr}$ & $\mathrm{ml}$ & $\mathrm{P}$ & Sayedur665 \\
\hline V. sativa $\mathrm{L}$. & Bonmoshur & NMS & Herb, c & $\mathrm{Oz} ; \mathrm{Sr}$ & $\mathrm{ml}$ & $\mathrm{W}$ & Sayedur213 \\
\hline $\begin{array}{l}\text { "Vigna adenantha (G. Mey.) } \\
\text { Maréchal }\end{array}$ & Bon borboti & NMS & Herb, c & $\mathrm{Mz} ; \mathrm{Sr}, \mathrm{Kh}$ & $\mathrm{ml}$, os & $\mathrm{W}$ & Sayedur1179 \\
\hline${ }^{*} V$. luteola (Jacq.) Benth. & Holdeymug & NMS & Herb, c & $\mathrm{Oz}, \mathrm{Mz} ; \mathrm{Sr}, \mathrm{Ch}$ & $\mathrm{ml}$ & $\mathrm{W}$ & Sayedur1188 \\
\hline V. marina (Burm.) Merr. & Nonta shim & NMS & Herb, c & $\mathrm{Pz} ; \mathrm{St}$ & $\mathrm{ml}$ & $\mathrm{W}$ & N1300 \\
\hline V. radiata (L.) R. Wilczek. & Hanimug & NMS & Herb, c & $\mathrm{Mz} ; \mathrm{Sr}$ & $\mathrm{ml}$, os & $\mathrm{W}$ & N4688 \\
\hline${ }^{*} V$. trilobata (L.) Verdc. & Mugani & NMS & Herb, c & $\mathrm{Oz}, \mathrm{Mz} ; \mathrm{Sr}, \mathrm{Kh}$ & $\mathrm{ml}$ & $\mathrm{W}$ & Sayedur87 \\
\hline Zornia reticulata Sm. & Unknown & NMS & Herb, e & $\mathrm{Mz} ; \mathrm{Sr}$ & $\mathrm{ml}$ & $\mathrm{W}$ & Sayedur500 \\
\hline \multicolumn{8}{|l|}{ Sonneratiaceae Engl. } \\
\hline $\begin{array}{l}\text { "Sonneratia apetala Buch.- } \\
\text { Ham. }\end{array}$ & Kewra & MS & Tree, 1 & $\mathrm{Az} ; \mathrm{Ar}$ & $\mathrm{rb}, \mathrm{wd}$ & $\mathrm{Fr}, \mathrm{M}$ & Sayedur851 \\
\hline${ }^{*}$ S. caseolaris (L.) Engl. & Choila/ora & MS & Tree, $\mathrm{m}$ & $\mathrm{Oz}, \mathrm{Mz} ; \mathrm{Sr}, \mathrm{Ch}$ & $\mathrm{rb}$ & Fr, M & Sayedur229 \\
\hline \multicolumn{8}{|l|}{ Lythraceae J. St.-Hil. } \\
\hline *Ammannia verticillata Lam. & Niatai & NMS & Herb, e & $\mathrm{Oz}, \mathrm{Mz} ; \mathrm{Sr}$ & wt & $\mathrm{W}$ & Sayedur1317 \\
\hline $\begin{array}{l}\text { Lagerstroemia speciosa (L.) } \\
\text { Pers. }\end{array}$ & Jarul & NMS & Tree, $\mathrm{m}$ & $\mathrm{Oz} ; \mathrm{Sr}$ & $w d, r b$ & $\mathrm{~T}, \mathrm{O}$ & Sayedur611 \\
\hline Lawsonia inermis L. & Mehedi & NMS & Shrub & $\mathrm{Az} ; \mathrm{Ar}$ & $\mathrm{ml}(\mathrm{pl})$ & M, D & Sayedur916 \\
\hline Rotala indica (Willd.) Koehne & Deshi ghurni & NMS & Herb, e & $\mathrm{Mz} ; \mathrm{Sr}$ & wt & $\mathrm{W}$ & Sayedur701 \\
\hline \multicolumn{8}{|l|}{ Myrtaceae Juss. } \\
\hline $\begin{array}{l}\text { Callistemon citrinus (Curtis) } \\
\text { Skeels }\end{array}$ & Bottle brash & NMS & Shrub & $\mathrm{Az} ; \mathrm{Ar}$ & $\mathrm{ml}(\mathrm{pl})$ & $\mathrm{O}$ & Sayedur38 \\
\hline${ }^{*}$ Psidium guajava $\mathrm{L}$. & Pyera & NMS & Tree, $\mathrm{s}$ & $\mathrm{Az} ; \mathrm{Ar}$ & $\mathrm{ml}(\mathrm{pl})$ & $\mathrm{Fr}$ & Sayedur621 \\
\hline Syzygium cumini (L.) Skeels & Kalo jam & NMS & Tree, 1 & $\mathrm{Az} ; \mathrm{Ar}$ & $\mathrm{ml}(\mathrm{pl})$ & $\mathrm{Fr}, \mathrm{T}$ & Sayedur1043 \\
\hline "S. fruticosum Roxb. ex DC. & Khudi jam & NMS & Tree, $\mathrm{m}$ & $\mathrm{Oz}, \mathrm{Mz} ; \mathrm{Sr}, \mathrm{Ch}$ & $\mathrm{ml}, \mathrm{os}$ & $\mathrm{Fr}, \mathrm{T}$ & Sayedur891 \\
\hline \multicolumn{8}{|l|}{ Onagraceae Juss. } \\
\hline $\begin{array}{l}\text { Ludwigia adscendens (L.) H. } \\
\text { Hara }\end{array}$ & Keshordom & NMS & Herb, a & $\mathrm{Az} ; \mathrm{Ar}$ & wt & $\mathrm{W}$ & Sayedur1113 \\
\hline L. hyssopifolia (G. Don) Exell & Panilong & NMS & Herb, e & $\mathrm{Az} ; \mathrm{Ar}$ & wt & $\mathrm{W}$ & Sayedur161 \\
\hline L. perennis L. & Amorkura & NMS & Herb, e & $\mathrm{Mz} ; \mathrm{Sr}$ & wt & $\mathrm{W}$ & N1237 \\
\hline \multicolumn{8}{|l|}{ Combretaceae R. Br. } \\
\hline Combretum acuminatum Roxb. & Patuinia & NMS & Herb, c & $\mathrm{Oz} ; \mathrm{Sr}$ & wd & $\mathrm{W}$ & Sayedur290 \\
\hline *Lumnitzera racemosa Willd. & Kirpa/cirpa & MS & Tree, $\mathrm{s}$ & $\mathrm{Pz} ; \mathrm{St}$ & $\mathrm{wd}, \mathrm{fm}$ & $\mathrm{M}, \mathrm{Fw}$ & Sayedur462 \\
\hline $\begin{array}{l}\text { Terminalia arjuna (Roxb. ex } \\
\text { DC.) Wight \& Arn. }\end{array}$ & Arjun & NMS & Tree, $\mathrm{m}$ & $\mathrm{Az} ; \mathrm{Ar}$ & $\mathrm{ml}(\mathrm{pl})$ & M & Sayedur1144 \\
\hline
\end{tabular}


Table 2. Contd.

\begin{tabular}{|c|c|c|c|c|c|c|c|}
\hline Scientific name & Bangla name & $\mathrm{MC}$ & Habit & Distribution & Habitat & Use & $\mathrm{RS}$ \\
\hline T. belerica (Gaertn.) Roxb. & Bohera & NMS & Tree, 1 & $\mathrm{Az} ; \mathrm{Ar}$ & $\mathrm{ml}(\mathrm{pl})$ & $\mathrm{M}, \mathrm{T}$ & Sayedur818 \\
\hline T. catappa $\mathrm{L}$. & Kathbadam & MAS & Tree, 1 & $\mathrm{Az} ; \mathrm{Ar}$ & $\mathrm{ml}(\mathrm{pl})$ & Fw, Fr & Sayedur1148 \\
\hline \multicolumn{8}{|l|}{ Rhizophoraceae Pers. } \\
\hline${ }^{*}$ Bruguiera gymnorhiza (L.) Savigny & Kakra & MS & Tree, 1 & $\mathrm{Mz}, \mathrm{Pz} ; \mathrm{Kh}, \mathrm{St}$ & wd & $\mathrm{T}$ & Sayedur416 \\
\hline${ }^{*}$ B. sexangula (Lour.) Poir. & Lal kakra & MS & Tree, $\mathrm{m}$ & $\mathrm{Mz}, \mathrm{Pz} ; \mathrm{Ch}, \mathrm{St}$ & wd & $\mathrm{T}, \mathrm{FW}$ & Sayedur60 \\
\hline${ }^{*}$ Ceriops decandra (Griff.) W. Theob. & Goran & MS & Shrub & $\mathrm{Az} ; \mathrm{Ar}$ & wd & $\mathrm{M}, \mathrm{FW}_{\mathrm{W}}$ & Sayedur1119 \\
\hline${ }^{*}$ Kandelia candel (L.) Druce & Bhatkathi & MS & Shrub & $\mathrm{Mz}, \mathrm{Pz} ; \mathrm{Kh}, \mathrm{St}$ & $\mathrm{fm}, \mathrm{rb}$ & $\mathrm{M}, \mathrm{Fw}$ & Sayedur885 \\
\hline${ }^{*}$ Rhizophora apiculata Blume & Bhorajhana & MS & Tree, $\mathrm{m}$ & $\mathrm{Mz}, \mathrm{Pz} ; \mathrm{Kh}, \mathrm{St}$ & $\mathrm{fm}, \mathrm{rb}$ & $\mathrm{M}, \mathrm{FW}$ & Sayedur53 \\
\hline${ }^{*} R$. mucronata Lam. & Jhana & MS & Tree, $\mathrm{m}$ & $\mathrm{Mz}, \mathrm{Pz} ; \mathrm{Kh}, \mathrm{St}$ & $\mathrm{fm}, \mathrm{rb}$ & $\mathrm{M}, \mathrm{Fw}$ & Sayedur37 \\
\hline \multicolumn{8}{|l|}{ Loranthaceae Juss. } \\
\hline Dendrophthoe falcata Ettingsh. & Porgacha & MAS & Shrub, ps & $\mathrm{Az} ; \mathrm{Ar}$ & tb & $\mathrm{W}$ & Sayedur408 \\
\hline $\begin{array}{l}\text { Macrosolen cochinchinensis (Lour.) } \\
\text { Tiegh. }\end{array}$ & Porgacha & MAS & Shrub, ps & $\mathrm{Az} ; \mathrm{Ar}$ & $\mathrm{tb}$ & $\mathrm{W}$ & Sayedur351 \\
\hline "Scurrula parasitica L. & Porgacha & NMS & Shrub, ps & $\mathrm{Az} ; \mathrm{Ar}$ & $\mathrm{tb}$ & $\mathrm{W}$ & Sayedur387 \\
\hline *Viscum monoicum Roxb. ex DC. & Banda & MAS & Shrub, ps & $\mathrm{Az} ; \mathrm{Ar}$ & $\mathrm{tb}$ & $\mathrm{W}$ & Sayedur1941 \\
\hline \multicolumn{8}{|l|}{ Celastraceae R. Br. } \\
\hline "Salacia chinensis L. & Choita boroi & MAS & Shrub, sc & $\mathrm{Az} ; \mathrm{Ar}$ & wd, fm & Fr, M & Sayedur442 \\
\hline \multicolumn{8}{|l|}{ Euphorbiaceae Juss. } \\
\hline "Acalypha indica L. & Muktajhuri & NMS & Herb, e & $\mathrm{Oz} ; \mathrm{Sr}$ & $\mathrm{ml}$ & M & Mosharof5 \\
\hline Bischofia javanica Blume. & Kainjal & NMS & Tree, $\mathrm{m}$ & $\mathrm{Oz} ; \mathrm{Sr}$ & $\mathrm{rb}$ & $\mathrm{Fw}$ & Sayedur329 \\
\hline Breynia retusa (Dennst.) Alston & Silpati & NMS & Shrub & $\mathrm{Oz} ; \mathrm{Sr}$ & os, $\mathrm{ml}$ & W & Sayedur466 \\
\hline${ }^{*}$ B. vitis-idaea (Burm. f.) C.E.C. Fisch. & Vita salpoti & NMS & Shrub & $\mathrm{Oz} ; \mathrm{Sr}$ & os, $\mathrm{ml}$ & $\mathrm{W}$ & Sayedur 725 \\
\hline${ }^{*}$ Bridelia stipularis (L.) Blume & Harinhara & NMS & Shrub & $\mathrm{Oz} ; \mathrm{Sr}$ & os, $\mathrm{ml}$ & $\mathrm{W}$ & Sayedur953 \\
\hline $\begin{array}{l}\text { *Chrozophora oblongifolia (Del.) Adr. } \\
\text { Juss. ex Spreng. }\end{array}$ & Khudiphora & NMS & Herb, e & $\mathrm{Mz} ; \mathrm{Sr}$ & $\mathrm{ml}$ & $\mathrm{W}$ & Sayedur648 \\
\hline${ }^{*}$ C. plicata (Vahl) A. Juss. ex Spreng. & Khudiokra & NMS & Herb, e & $\mathrm{Oz} ; \mathrm{Sr}$ & $\mathrm{ml}$ & $\mathrm{W}$ & Sayedur1229 \\
\hline Croton bonplandianus Baill. & Bon croton & NMS & Herb, e & $\mathrm{Oz} ; \mathrm{Sr}$ & $\mathrm{ml}, \mathrm{gl}$ & $\mathrm{W}$ & Sayedur512 \\
\hline C. caudatus Geiseler. & Nanbhantur & NMS & Shrub & $\mathrm{Oz} ; \mathrm{Sr}$ & os & $\mathrm{W}$ & Mosharof66 \\
\hline C. tiglium L. & Jamalgota & NMS & Herb, e & $\mathrm{Mz} ; \mathrm{Sr}$ & $\mathrm{ml}$ & $\mathrm{W}$ & Sayedur509 \\
\hline $\begin{array}{l}{ }^{*} \text { Drypetes assamica (Hook. f.) Pax \& } \\
\text { K. Hoffm. }\end{array}$ & Bon bokul & NMS & Shrub & $\mathrm{Mz} ; \mathrm{Sr}$ & os & Fw & Sayedur688 \\
\hline${ }^{*}$ Euphorbia hirta L. & Dudhiya & NMS & Herb, $p$ & $\mathrm{Az} ; \mathrm{Ar}$ & $\mathrm{ml}$ & $\mathrm{W}, \mathrm{M}$ & Sayedur547 \\
\hline E. hispida Boiss. & Lomahori & NMS & Herb, $p$ & $\mathrm{Az} ; \mathrm{Sr}, \mathrm{Kh}$ & $\mathrm{ml}$ & W & Sayedur1013 \\
\hline E. prostrata Aiton & Sijhori & NMS & Herb, $p$ & $\mathrm{Pz} ; \mathrm{Kh}$ & $\mathrm{ml}$ & $\mathrm{W}$ & Sayedur2169 \\
\hline E. serpens Kunth & Balumadur & NMS & Herb, $p$ & $\mathrm{Pz} ; \mathrm{Kh}$ & $\mathrm{ml}$ & $\mathrm{W}$ & Sayedur28 \\
\hline${ }^{*}$ E. thymifolia L. & Swetkerui & NMS & Herb, $p$ & $\mathrm{Mz}, \mathrm{Pz} ; \mathrm{Sr}, \mathrm{Kh}$ & $\mathrm{ml}$ & $\mathrm{W}$ & Sayedur849 \\
\hline${ }^{*}$ Excoecaria agallocha $\mathrm{L}$. & Gewa & MS & Tree, $\mathrm{m}$ & $\mathrm{Az} ; \mathrm{Ar}$ & wd & $\mathrm{M}, \mathrm{Sw}$ & Sayedur429 \\
\hline $\begin{array}{l}\text { Flueggea virosa (Roxb. ex Willd.) } \\
\text { Voigt }\end{array}$ & Chitka lata & NMS & Shrub & $\mathrm{Oz} ; \mathrm{Sr}$ & $\mathrm{ml}, \mathrm{rb}$ & W & Sayedur775 \\
\hline $\begin{array}{l}{ }^{*} \text { Mallotus repandus (Rottler) Müll. } \\
\text { Arg. }\end{array}$ & Gunti & NMS & Shrub & $\mathrm{Oz}, \mathrm{Pz} ; \mathrm{Sr}, \mathrm{Kh}$ & $\mathrm{ml}$ & $\mathrm{W}$ & Sayedur647 \\
\hline Pedilanthus tithymaloides Poit. & Chitta & NMS & Herb, e & $\mathrm{Oz} ; \mathrm{Sr}$ & $\mathrm{ml}$ & $\mathrm{O}$ & Sayedur 2184 \\
\hline
\end{tabular}


Table 2. Contd.

\begin{tabular}{|c|c|c|c|c|c|c|c|}
\hline Scientific name & Bangla name & $\mathrm{MC}$ & Habit & Distribution & Habitat & Use & RS \\
\hline Phyllanthus emblica L. & Amloki & NMS & Tree, $\mathrm{s}$ & $\mathrm{Az} ; \mathrm{Ar}$ & $\mathrm{ml}, \mathrm{os}$ & Fr, M & Sayedur687 \\
\hline${ }^{*} P$. niruri L. & Bhuiamla & NMS & Herb, e & $\mathrm{Az} ; \mathrm{Ar}$ & $\mathrm{ml}, \mathrm{gl}$ & W & Sayedur1091 \\
\hline P. reticulatus Poir. & Chitka & NMS & Shrub & $\mathrm{Oz} ; \mathrm{Sr}$ & $\mathrm{ml}$, os & $\mathrm{W}$ & Sayedur907 \\
\hline P. urinaria $\mathrm{L}$. & Kalochitki & NMS & Shrub & $\mathrm{Oz}, \mathrm{Pz} ; \mathrm{Sr}, \mathrm{Kh}$ & $\mathrm{ml}$ & $\mathrm{W}$ & Sayedur485 \\
\hline P. virgatus G. Forst. & Chitki & NMS & Shrub & $\mathrm{Oz}, \mathrm{Pz} ; \mathrm{Sr}, \mathrm{Kh}$ & $\mathrm{ml}$ & $\mathrm{W}$ & Sayedur1088 \\
\hline Ricinus communis L. & Venna/reri & MAS & Shrub & $\mathrm{Oz} ; \mathrm{Sr}$ & $\mathrm{ml}$, os & M, Oi & Sayedur823 \\
\hline "Shirakiopsis indica (Willd.) Esser & Hurmui & MAS & Tree, $\mathrm{s}$ & $\mathrm{Oz}, \mathrm{Mz} ; \mathrm{Sr}, \mathrm{Kh}$ & $\mathrm{wd}, \mathrm{rb}$ & $\mathrm{M}, \mathrm{P}$ & Sayedur671 \\
\hline Suregada multiflora (A. Juss.) Baill. & Bob naringa & NMS & Shrub & $\mathrm{Oz} ; \mathrm{Sr}$ & os, fm & $\mathrm{Fw}$ & Sayedur673 \\
\hline *Trewia polycarpa Benth. \& Hook.f. & Pitali & NMS & Tree, $\mathrm{m}$ & $\mathrm{Oz}, \mathrm{Mz} ; \mathrm{Sr}$ & $\mathrm{ml}$, os & $\mathrm{T}, \mathrm{Fw}$ & Sayedur2005 \\
\hline \multicolumn{8}{|l|}{ Rhamnaceae Juss. } \\
\hline Colubrina javanica Miq. & Bon boroi & NMS & Shrub, s & $\mathrm{Mz} ; \mathrm{Sr}$ & os & M & Sayedur1081 \\
\hline "Ziziphus oenopolia (L.) Mill. & Bon boroi & NMS & Shrub, s & $\mathrm{Oz} ; \mathrm{Sr}$ & os, wd & $\mathrm{Fw}$ & Sayedur 672 \\
\hline${ }^{*} Z$. mauritiana Lam. & Boroi & NMS & Tree, s & $\mathrm{Az} ; \mathrm{Ar}$ & $\mathrm{ml}, \mathrm{os}$ & Fr, Fw & Sayedur3093 \\
\hline \multicolumn{8}{|l|}{ Leeaceae Dumort. } \\
\hline "Leea indica (Burm. f.) Merr. & Kakjangha & NMS & Shrub & $\mathrm{Oz} ; \mathrm{Sr}$ & $\mathrm{fm}, \mathrm{rb}$ & $\mathrm{M}, \mathrm{W}$ & Sayedur1258 \\
\hline \multicolumn{8}{|l|}{ Vitaceae Juss. } \\
\hline "Ampelocissus latifolia (Roxb.) Planch. & Unknown & NMS & Herb, c & $\mathrm{Oz}, \mathrm{Mz} ; \mathrm{Sr}$ & $\mathrm{fm}, \mathrm{os}$ & $\mathrm{W}$ & Sayedur810 \\
\hline Cissus assamica (M.A. Lawson) Craib. & Angurlata & NMS & Herb, c & $\mathrm{Oz}, \mathrm{Mz} ; \mathrm{Sr}$ & $\mathrm{rb}, \mathrm{os}$ & W & Sayedur1031 \\
\hline Cayratia japonica (Thunb.) Gagnep. & Golgotilata & NMS & Herb, c & $\mathrm{Oz} ; \mathrm{Sr}$ & $\mathrm{fm}, \mathrm{os}$ & $\mathrm{W}$ & Sayedur323 \\
\hline${ }^{*}$ C. trifolia (L.) Domin & Angurlata & NMS & Herb, c & $\mathrm{Az} ; \mathrm{Ar}$ & $\mathrm{fm}, \mathrm{os}$ & M & Sayedur949 \\
\hline \multicolumn{8}{|l|}{ Polygalaceae Hoffmanns. \& Link } \\
\hline Polygala chinensis L. & Meradu & NMS & Herb, $p$ & $\mathrm{Oz}, \mathrm{Pz} ; \mathrm{Sr}, \mathrm{Kh}$ & $\mathrm{ml}$ & $\mathrm{W}$ & Sayedur1191 \\
\hline P. erioptera DC. & Teradudhi & NMS & Herb, $p$ & $\mathrm{Pz} ; \mathrm{Kh}$ & $\mathrm{ml}$ & $\mathrm{W}$ & Sayedur162 \\
\hline \multicolumn{8}{|l|}{ Sapindaceae Juss. } \\
\hline “Allophylus cobbe (L.) Raeusch. & Chita & MAS & Shrub & $\mathrm{Oz} ; \mathrm{Sr}$ & os & $\mathrm{W}$ & Sayedur613 \\
\hline "Cardiospermum halicacabum L. & Phutki & NMS & Herb, c & $\mathrm{Oz} ; \mathrm{Sr}$ & $\mathrm{ml}$, os & M & Sayedur1152 \\
\hline Dodonaea viscosa Jacq. & Paniaphul & NMS & Tree, $\mathrm{s}$ & $\mathrm{Mz}, \mathrm{Pz} ; \mathrm{Sr}, \mathrm{St}$ & fm, wd & $\mathrm{M}, \mathrm{Fw}$ & Sayedur1250 \\
\hline Lepisanthes rubiginosa (Roxb.) Leenh. & Horina & NMS & Shrub & $\mathrm{Oz} ; \mathrm{Sr}$ & fm, wd & Fr, M & Sayedur811 \\
\hline L. senegalensis (Juss. ex Poir.) Leenh. & Gotahorina & NMS & Shrub & $\mathrm{Oz} ; \mathrm{Sr}$ & $\mathrm{fm}$ & $\mathrm{W}$ & Sayedur296 \\
\hline \multicolumn{8}{|l|}{ Anacardiaceae R. Br. } \\
\hline${ }^{*}$ Lannea coromandelica (Houtt.) Merr. & Jiga/jeol & NMS & Tree, $\mathrm{s}$ & $\mathrm{Oz}, \mathrm{Mz} ; \mathrm{Sr}$ & os, ml & $\mathrm{M}, \mathrm{Fw}$ & Sayedur902 \\
\hline Mangifera indica L. & Aam & NMS & Tree, $\mathrm{m}$ & $\mathrm{Az} ; \mathrm{Ar}$ & $\mathrm{ml}(\mathrm{pl})$ & $\mathrm{Fr}, \mathrm{T}$ & Sayedur2227 \\
\hline Spondias pinnata (L. f.) Kurz & Boonoamra & NMS & Tree, $\mathrm{m}$ & $\mathrm{Mz} ; \mathrm{Sr}$ & $\mathrm{fm}$ & $\mathrm{M}, \mathrm{Fw}$ & Sayedur1202 \\
\hline \multicolumn{8}{|l|}{ Meliaceae Juss. } \\
\hline Aglaia cucullata (Roxb.) Pellegr. & Amur & MS & Tree, $\mathrm{s}$ & $\mathrm{Az} ; \mathrm{Ar}$ & $\mathrm{wd}, \mathrm{rb}$ & $\mathrm{T}, \mathrm{FW}$ & Sayedur424 \\
\hline $\begin{array}{l}\text { Aphanamixis polystachya (Wall.) R. } \\
\text { Parker. }\end{array}$ & Roina/pitraj & NMS & Tree, s & $\mathrm{Oz} ; \mathrm{Sr}$ & fm, os & $\mathrm{M}, \mathrm{Fw}$ & Sayedur344 \\
\hline Azadirachta indica A. Juss. & Nim & NMS & Tree, $\mathrm{m}$ & $\mathrm{Az} ; \mathrm{Ar}$ & $\mathrm{ml}$ & $\mathrm{M}, \mathrm{T}$ & Sayedur1017 \\
\hline Swietenia mahagoni (L.) Jacq. & Mehagoni & NMS & Tree, 1 & $\mathrm{Az} ; \mathrm{Ar}$ & $\mathrm{ml}(\mathrm{pl})$ & $\mathrm{T}$ & Sayedur 2228 \\
\hline Toona ciliata M. Roem. & Tun & NMS & Tree, 1 & $\mathrm{Oz} ; \mathrm{Sr}$ & $\mathrm{ml}$, wd & $\mathrm{T}$ & Sayedur1049 \\
\hline
\end{tabular}


Table 2. Contd.

\begin{tabular}{|c|c|c|c|c|c|c|c|}
\hline Scientific name & Bangla name & $\mathrm{MC}$ & Habit & Distribution & Habitat & Use & $\mathrm{RS}$ \\
\hline $\begin{array}{l}{ }^{*} \text { Xylocarpus granatum J. } \\
\text { Koenig }\end{array}$ & Dhundal & MS & Tree, $\mathrm{m}$ & $\mathrm{Mz}, \mathrm{Pz} ; \mathrm{Kh}, \mathrm{St}$ & $\mathrm{rb}, \mathrm{wd}$ & $\mathrm{T}, \mathrm{M}$ & Sayedur1120 \\
\hline $\begin{array}{l}{ }^{*} \text { X. moluccensis (Lam.) M. } \\
\text { Roem. }\end{array}$ & Poshur & MS & Tree, 1 & $\mathrm{Az} ; \mathrm{Ar}$ & wd & $\mathrm{T}, \mathrm{M}$ & Sayedur417 \\
\hline \multicolumn{8}{|l|}{ Rutaceae Juss. } \\
\hline${ }^{*}$ Aegle marmelos (L.) Corrêa & Bel & NMS & Tree, $\mathrm{m}$ & $\mathrm{Az} ; \mathrm{Ar}$ & $\mathrm{ml}(\mathrm{pl})$ & $\mathrm{M}, \mathrm{Fr}$ & Sayedur1149 \\
\hline Citrus maxima (Burm.) Merr. & Jambura & NMS & Tree, $\mathrm{s}$ & $\mathrm{Az} ; \mathrm{Ar}$ & $\mathrm{ml}(\mathrm{pl})$ & $\mathrm{Fr}$ & Sayedur1291 \\
\hline C. medica $\mathrm{L}$. & Panilebu & NMS & Shrub & $\mathrm{Mz} ; \mathrm{Sr}$ & $\mathrm{ml}$ & $\mathrm{Fr}$ & Sayedur 1290 \\
\hline Feronia limonia (L.) Swingle & Kodbel & NMS & Tree, $\mathrm{m}$ & Pz; St & $\mathrm{ml}(\mathrm{pl})$ & $\mathrm{Fr}$ & Sayedur223 \\
\hline $\begin{array}{l}\text { *Glycosmis pentaphylla (Retz.) } \\
\text { DC. }\end{array}$ & Motkila & NMS & Shrub & $\mathrm{Oz}, \mathrm{Mz} ; \mathrm{Sr}$ & $\mathrm{os}, \mathrm{ml}$ & $\mathrm{M}, \mathrm{Fw}$ & Sayedur294 \\
\hline $\begin{array}{l}{ }^{*} \text { Merope angulata (Willd.) } \\
\text { Swingle }\end{array}$ & Bonlebu & MAS & Shrub & $\mathrm{Oz}, \mathrm{Mz} ; \mathrm{Sr}$ & $\mathrm{fm}$ & $\mathrm{W}$ & Sayedur646 \\
\hline \multicolumn{8}{|l|}{ Oxalidaceae R. Br. } \\
\hline $\begin{array}{l}\text { *Oxalis corniculata L. } \\
\text { Apiaceae Lindl. }\end{array}$ & Amrul & $\begin{array}{l}\text { NMS } \\
\text { NMS }\end{array}$ & Herb, $p$ & $\mathrm{Oz} ; \mathrm{Sr}$ & $\mathrm{ml}, \mathrm{gl}$ & $\mathrm{W}, \mathrm{M}$ & Sayedur1297 \\
\hline Centella asiatica (L.) Urb. & Thankuni & NMS & Herb, cr & $\mathrm{Az} ; \mathrm{Ar}$ & $\mathrm{ml}, \mathrm{gl}$ & M & Sayedur754 \\
\hline Eryngium foetidum L. & Bilatedhoniya & NMS & Herb, e & $\mathrm{Az} ; \mathrm{Ar}$ & $\mathrm{ml}(\mathrm{cl})$ & $\mathrm{V}, \mathrm{Sp}$ & Sayedur946 \\
\hline $\begin{array}{l}\text { Oenanthe benghalensis (Roxb.) } \\
\text { Kurz }\end{array}$ & Bondhoniya & NMS & Herb, e & $\mathrm{Oz} ; \mathrm{Sr}$ & $\mathrm{ml}$ & $\mathrm{W}$ & Sayedur 1279 \\
\hline O. thomsonii C.B. Clarke & Unknown & NMS & Herb, e & $\mathrm{Oz} ; \mathrm{Sr}$ & $\mathrm{ml}$ & $\mathrm{W}$ & Sayedur2166 \\
\hline $\begin{array}{l}\text { Pimpinella heyneana (DC.) } \\
\text { Benth. }\end{array}$ & Pimpin & NMS & Herb, e & $\mathrm{Oz} ; \mathrm{Sr}$ & $\mathrm{ml}$ & $\mathrm{W}$ & Sayedur1314 \\
\hline \multicolumn{8}{|l|}{ Gentianaceae Juss. } \\
\hline \multicolumn{7}{|l|}{ Apocynaceae Juss. } & Sayedur707 \\
\hline Alstonia scholaris (L.) R. Br. & Chatim & NMS & Tree, $\mathrm{m}$ & $\mathrm{Oz} ; \mathrm{Sr}$ & $\mathrm{ml}, \mathrm{fm}$ & M & Sayedur709 \\
\hline $\begin{array}{l}\text { Catharanthus roseus (L.) G. } \\
\text { Don }\end{array}$ & Nayantara & NMS & Herb, e & $\mathrm{Oz}, \mathrm{Mz} ; \mathrm{Sr}$ & $\mathrm{ml}, \mathrm{os}$ & M & Sayedur649 \\
\hline "Cerbera odollam Gaertn. & Dahur/dakur & MAS & Tree, $\mathrm{s}$ & $\mathrm{Oz}, \mathrm{Mz} ; \mathrm{Sr}, \mathrm{Ch}$ & $\mathrm{rb}, \mathrm{wd}$ & M & Sayedur117 \\
\hline $\begin{array}{l}\text { Ichnocarpus frutescens (L.) } \\
\text { W.T. Aiton }\end{array}$ & Dudhia lata & NMS & Herb, c & $\mathrm{Oz} ; \mathrm{Sr}$ & os & M & Mosharof118 \\
\hline $\begin{array}{l}{ }^{*} \text { Parsonsia alboflavescens } \\
\text { (Dennst.) Mabb. }\end{array}$ & Pasonsi & NMS & Herb, c & $\mathrm{Az} ; \mathrm{Ar}$ & $\mathrm{fm}, \mathrm{rb}$ & $\mathrm{W}$ & Sayedur216 \\
\hline $\begin{array}{l}\text { Tabernaemontana divaricata } \\
\text { (L.) R. Br. ex Roem. \& Schult }\end{array}$ & Togor & NMS & Shrub & $\mathrm{Mz} ; \mathrm{Ch}$ & $\mathrm{ml}(\mathrm{pl})$ & $\mathrm{O}, \mathrm{M}$ & Sayedur350 \\
\hline $\begin{array}{l}\text { Thevetia peruviana (Pers.) K. } \\
\text { Schum. }\end{array}$ & Kolkeyful & NMS & Tree, $\mathrm{s}$ & $\mathrm{Mz}, \mathrm{Pz} ; \mathrm{Kh}, \mathrm{St}$ & $\mathrm{ml}(\mathrm{pl})$ & M & Sayedur878 \\
\hline \multicolumn{8}{|l|}{ Asclepiadaceae Borkh. } \\
\hline $\begin{array}{l}{ }^{*} \text { Calotropis gigantea (L.) W.T. } \\
\text { Aiton }\end{array}$ & Akond & MAS & Shrub & $\mathrm{Oz}, \mathrm{Mz} ; \mathrm{Sr}$ & $\mathrm{ml}$ & M & Sayedur653 \\
\hline C. procera (Aiton) W.T. Aiton & Swetakond & NMS & Shrub & $\mathrm{Oz}, \mathrm{Mz} ; \mathrm{Sr}$ & $\mathrm{ml}$ & M & Sayedur249 \\
\hline Ceropegia lucida Wall. & Lucipega & NMS & Herb, c & $\mathrm{Mz}, \mathrm{Pz} ; \mathrm{Sr}, \mathrm{St}$ & $\mathrm{fm}$ & $\mathrm{W}$ & Sayedur588 \\
\hline
\end{tabular}


Table 2. Contd.

\begin{tabular}{|c|c|c|c|c|c|c|c|}
\hline Scientific name & Bangla name & $\mathrm{MC}$ & Habit & Distribution & Habitat & Use & RS \\
\hline${ }^{*}$ Dregea volubilis (L.f.) Benth. ex Hook. f & Titakunja lata & NMS & Herb, c & $\mathrm{Oz}, \mathrm{Pz} ; \mathrm{Sr}, \mathrm{Kh}$ & $\mathrm{wd}, \mathrm{rb}$ & W & Sayedur1280 \\
\hline${ }^{*}$ Finlaysonia obovata Wall. & Mamakola & MAS & Herb, c & $\mathrm{Az} ; \mathrm{Ar}$ & $\mathrm{wd}, \mathrm{rb}$ & W & Sayedur440 \\
\hline *Hoya parasitica Wall. ex Wight & Futki lata & MAS & Herb, ps & $\mathrm{Oz}, \mathrm{Mz}, \mathrm{Sr}, \mathrm{Ch}, \mathrm{Kh}$ & $\mathrm{tt}$ & W & Sayedur400 \\
\hline${ }^{*}$ Pentatropis capensis (L.f.) Bullock & Panchpis & NMS & Herb, c & $\mathrm{Pz} ; \mathrm{Kh}$ & $\mathrm{fm}$ & W & Sayedur469 \\
\hline *Sarcolobus carinatus Wall. & Bawali lata & MAS & Herb, c & $\mathrm{Az} ; \mathrm{Ar}$ & wd, fm & M & Sayedur885 \\
\hline${ }^{*}$ S. globosus Wall. & Bawali lata & MAS & Herb, c & $\mathrm{Az} ; \mathrm{Ar}$ & wd, fm & M & Sayedur888 \\
\hline Tylophora indica (Burm. f.) Merr. & Anatamul & NMS & Herb, c & $\mathrm{Mz}, \mathrm{Pz} ; \mathrm{Sr}, \mathrm{Kh}$ & $\mathrm{fm}, \mathrm{ml}$ & M & Sayedur630 \\
\hline *T. tenuissima (Roxb.) Wight \& Arn. & Nishimul & NMS & Herb, c & $\mathrm{Pz} ; \mathrm{Kh}$ & $\mathrm{fm}, \mathrm{ml}$ & $\mathrm{W}$ & Sayedur1007 \\
\hline \multicolumn{8}{|l|}{ Solanaceae Juss. } \\
\hline Capsicum frutescens $\mathrm{L}$. & Morich & NMS & Shrub & $\mathrm{Az} ; \mathrm{Ar}$ & $\mathrm{ml}(\mathrm{pl})$ & $\mathrm{Sp}$ & Sayedur1169 \\
\hline Datura metel L. & Dhutra & NMS & Shrub & $\mathrm{Oz} ; \mathrm{Sr}$ & $\mathrm{ml}$, os & M & Sayedur1302 \\
\hline Nicotiana plumbaginifolia Viv. & Bantamak & NMS & Herb, e & $\mathrm{Oz} ; \mathrm{Sr}$ & $\mathrm{ml}$ & W & Sayedur72 \\
\hline Physalis minima $\mathrm{L}$. & Kapalphutki & NMS & Herb, e & $\mathrm{Oz} ; \mathrm{Sr}$ & $\mathrm{ml}$ & M & Sayedur586 \\
\hline Solanum aculeatissimum Jacq. & Akuli begun & NMS & Herb, $p$ & $\mathrm{Oz} ; \mathrm{Sr}$ & $\mathrm{ml}, \mathrm{os}$ & W & Sayedur2205 \\
\hline S. americanum Mill. & Tit begub & NMS & Herb, e & $\mathrm{Oz} ; \mathrm{Sr}$ & $\mathrm{ml}$, os & W & Sayedur1323 \\
\hline S. violaceum Ortega & Phuti begun & NMS & Shrub & $\mathrm{Oz} ; \mathrm{Sr}$ & $\mathrm{ml}, \mathrm{os}$ & $\mathrm{M}, \mathrm{V}$ & Sayedur2111 \\
\hline *S. nigrum L. & Kakmachi & NMS & Herb, e & $\mathrm{Oz} ; \mathrm{Sr}$ & $\mathrm{ml}$ & W & Sayedur215 \\
\hline S. sisymbrifolium Lam. & Kata begun & NMS & Herb, $p$ & $\mathrm{Oz}, \mathrm{Mz} ; \mathrm{Sr}$ & $\mathrm{ml}$ & W & Mosharof199 \\
\hline S. torvum Sw. & Titbegun & NMS & Shrub & $\mathrm{Oz} ; \mathrm{Sr}$ & $\mathrm{ml}$, os & W & Sayedur339 \\
\hline "S. virginianum L. & Kantikari & NMS & Herb, $p$ & $\mathrm{Mz}, \mathrm{Pz} ; \mathrm{Sr}, \mathrm{St}$ & $\mathrm{sd}, \mathrm{fm}$ & W & Sayedur471 \\
\hline \multicolumn{8}{|l|}{ Convolvulaceae Juss. } \\
\hline Aniseia martinicensis (Jacq.) Choisy & Shadamati & NMS & Herb, c & $\mathrm{Pz} ; \mathrm{St}$ & $\mathrm{ml}$ & W & N1367 \\
\hline *Argyreia nervosa (Burm. f.) Bojer. & Bijtarak & NMS & Herb, c & $\mathrm{Oz} ; \mathrm{Sr}$ & $\mathrm{fm}, \mathrm{ml}$ & M & Mosharof22 \\
\hline Bonamia semidigyna (Roxb.) Hallier f. & Gandabhadi & NMS & Herb, c & $\mathrm{Mz} ; \mathrm{Sr}$ & $\mathrm{fm}, \mathrm{os}$ & W & Sayedur655 \\
\hline *Cuscuta reflexa Roxb. & Sharnalata & NMS & Herb, ps & $\mathrm{Az} ; \mathrm{Ar}$ & $\mathrm{pc}$ & $\mathrm{W}, \mathrm{M}$ & Sayedur853 \\
\hline Evolvulus nummularius (L.) L. & Khetpapra & NMS & Herb, cr & $\mathrm{Oz} ; \mathrm{Sr}$ & $\mathrm{ml}, \mathrm{gl}$ & W & Sayedur1087 \\
\hline Hewittia malabarica (L.) Suresh & Hiwet & NMS & Herb, c & $\mathrm{Oz}, \mathrm{Mz} ; \mathrm{Sr}$ & os, $\mathrm{ml}$ & W & Sayedur571 \\
\hline${ }^{*}$ Ipomoea aquatica Forssk. & Kolmishak & NMS & Herb, a & $\mathrm{Az} ; \mathrm{Ar}$ & wt & V & Sayedur26 \\
\hline I. batatas (L.) Lam. & Shakalu & NMS & Herb, cr & $\mathrm{Pz} ; \mathrm{Kh}$ & $\mathrm{ml}(\mathrm{pl})$ & $\mathrm{V}$ & Sayedur253 \\
\hline I. fistulosa Mart. ex Choisy & Dholkolmi & NMS & Shrub & $\mathrm{Oz}, \mathrm{Mz} ; \mathrm{Sr}, \mathrm{Kh}$ & $\mathrm{wt}, \mathrm{ml}$ & W & Mosharof121 \\
\hline I. littoralis Blume & Gang kolmi & NMS & Herb, c & $\mathrm{Mz} ; \mathrm{Sr}$ & $\mathrm{fm}$, os & $\mathrm{W}$ & N1378 \\
\hline I. marginata (Desr.) Verdc. & Bankalmi & MAS & Herb, $p$ & $\mathrm{Pz} ; \mathrm{Kh}$ & gl & $\mathrm{W}$ & Sayedur146 \\
\hline I. obscura (L.) Ker Gawl. & Kura kolmi & NAM & Herb, c & $\mathrm{Oz} ; \mathrm{Sr}$ & $\mathrm{ml}$ & W & Sayedur542 \\
\hline${ }^{*}$ I. pes-caprae (L.) R. Br. & Chagalkhuri & MAS & Herb, cr & $\mathrm{Mz} ; \mathrm{Sr}$ & sd & M & Mosharof43 \\
\hline Operculina turpethum (L.) S. Manso & Dudh kolmi & NMS & Herb, c & $\mathrm{Oz}, \mathrm{Mz} ; \mathrm{Sr}$ & $\mathrm{rb}, \mathrm{fm}$ & W & Sayedur745 \\
\hline "Stictocardia tiliifolia (Desr.) Hallier.f. & Bon kolmi & NMS & Herb, c & $\mathrm{Oz} ; \mathrm{Sr}$ & $\mathrm{fm}, \mathrm{ml}$ & $\mathrm{W}$ & Sayedur1271 \\
\hline \multicolumn{8}{|l|}{ Menyanthaceae Dumort. } \\
\hline "Nymphoides indicum (L.) Kuntze & Chandmala & NMS & Herb, a & $\mathrm{Oz}, \mathrm{Mz} ; \mathrm{Sr}, \mathrm{Ch}$ & wt & W & Sayedur172 \\
\hline \multicolumn{8}{|l|}{ Hydrophyllaceae R. Br. } \\
\hline *Hydrolea zeylanica (L.) Vahl. & Pani agra & NMS & Herb, a & $\mathrm{Mz} ; \mathrm{Sr}$ & wt & W & Sayedur1228 \\
\hline
\end{tabular}


Table 2. Contd.

\begin{tabular}{|c|c|c|c|c|c|c|c|}
\hline Scientific name & Bangla name & $\mathrm{MC}$ & Habit & Distribution & Habitat & Use & $\mathrm{RS}$ \\
\hline \multicolumn{8}{|l|}{ Boraginaceae Juss. } \\
\hline *Cordia dichotoma G. Forst. & Bohala/boula & MAS & Tree, $\mathrm{m}$ & $\mathrm{Oz}, \mathrm{Mz} ; \mathrm{Sr}$ & $\mathrm{fm}$, os & M & Sayedur461 \\
\hline Heliotropium curassavicum L. & Unknown & NMS & Herb, e & $\mathrm{Oz}, \mathrm{Mz} ; \mathrm{Sr}$ & $\mathrm{ml}$ & $\mathrm{W}$ & Sayedur1142 \\
\hline${ }^{*} H$. indicum L. & Hatishur & NMS & Herb, e & $\mathrm{Oz} ; \mathrm{Sr}$ & $\mathrm{ml}$ & M & Sayedur627 \\
\hline \multicolumn{8}{|l|}{ Verbenaceae J. St.-Hil. } \\
\hline "Avicennia marina (Forssk.) Vierh. & Morichabaen & MS & Tree, $\mathrm{m}$ & $\mathrm{Pz} ; \mathrm{Sr}, \mathrm{Kh}, \mathrm{St}$ & $\mathrm{rb}$ & $\mathrm{T}, \mathrm{M}$ & Sayedur410 \\
\hline${ }^{*}$ A. officinalis L. & Baen & MS & Tree, $\mathrm{m}$ & $\mathrm{Az} ; \mathrm{Ar}$ & wd, rb & $\mathrm{T}, \mathrm{M}$ & Sayedur52 \\
\hline${ }^{*}$ Clerodendrum indicum (L.) Kuntze & Bamunhati & NMS & Shrub & $\mathrm{Oz}, \mathrm{Mz} ; \mathrm{Sr}$ & $\mathrm{fm}$ & W & Sayedur1227 \\
\hline C. inerme (L.) Gaertn. & Sitka vat & MAS & Shrub & $\mathrm{Az} ; \mathrm{Ar}$ & $\mathrm{fm}$, os & $\mathrm{W}, \mathrm{M}$ & Sayedur1121 \\
\hline C. serratum Spreng. & Barangi & NMS & Shrub & $\mathrm{Oz} ; \mathrm{Sr}$ & $\mathrm{fm}, \mathrm{rb}$ & W & Sayedur222 \\
\hline C. viscosum Vent. & Vhat/ghetu & NMS & Shrub & $\mathrm{Oz}, \mathrm{Mz} ; \mathrm{Sr}$ & $\mathrm{ml}$, os & $\mathrm{W}, \mathrm{M}$ & Sayedur617 \\
\hline Clerodendrum sp. & Borositka vat & NMS & Shrub & $\mathrm{Oz}, \mathrm{Mz} ; \mathrm{Sr}, \mathrm{Kh}$ & $\mathrm{rb}$ & W & Sayedur1205 \\
\hline Gmelina arborea Roxb. & Gamari & NMS & Tree, $\mathrm{m}$ & $\mathrm{Oz}, \mathrm{Mz} ; \mathrm{Sr}, \mathrm{Kh}$ & $\mathrm{ml}(\mathrm{pl})$ & $\mathrm{T}$ & Sayedur999 \\
\hline $\begin{array}{l}{ }^{*} \text { Lippia alba (Mill.) N.E. Br. ex } \\
\text { Britton \& P. Wilson }\end{array}$ & Motmoti & NMS & Shrub & $\mathrm{Oz} ; \mathrm{Sr}$ & $\mathrm{ml}$ & $\mathrm{W}$ & Sayedur757 \\
\hline *Phyla nodiflora (L.) Greene & Bhuiokra & NMS & Herb, $p$ & $\mathrm{Oz} ; \mathrm{Sr}$ & $\mathrm{ml}$ & $\mathrm{W}$ & Sayedur838 \\
\hline *Premna serratifolia L. & Gambari & MAS & Shrub & $\mathrm{Oz}, \mathrm{Pz} ; \mathrm{Sr}, \mathrm{St}$ & $\mathrm{fm}, \mathrm{os}$ & M & Sayedur957 \\
\hline *Vitex negundo L. & Nishinda & NMS & Shrub & $\mathrm{Oz}, \mathrm{Pz} ; \mathrm{Sr}, \mathrm{St}$ & $\mathrm{fm}$, os & M & Sayedur484 \\
\hline \multicolumn{8}{|l|}{ Lamiaceae Martinov } \\
\hline *Anisomeles indica (L.) Kuntze & Gobura & NMS & Herb, e & $\mathrm{Mz}, \mathrm{Pz} ; \mathrm{Sr}, \mathrm{Kh}$ & $\mathrm{ml}$ & $\mathrm{W}$ & Sayedur26 \\
\hline Hyptis capitata Jacq. & Holkhusha & NMS & Herb, e & $\mathrm{Oz}, \mathrm{Pz} ; \mathrm{Sr}, \mathrm{Kh}$ & $\mathrm{ml}$, os & $\mathrm{W}$ & Sayedur1166 \\
\hline H. suaveolens (L.) Poit. & Tokma & NMS & Herb, e & $\mathrm{Mz}, \mathrm{Pz} ; \mathrm{Sr}, \mathrm{Kh}$ & $\mathrm{ml}$, os & M & Sayedur1151 \\
\hline Leucas aspera (Willd.) Link & Swetadran & NMS & Herb, e & $\mathrm{Oz}, \mathrm{Pz} ; \mathrm{Sr}, \mathrm{Kh}$ & $\mathrm{ml}, \mathrm{gl}$ & $\mathrm{W}$ & Sayedur103 \\
\hline${ }^{*}$ L. lavandulifolia Smith & Dondakolosh & NMS & Herb, e & $\mathrm{Pz} ; \mathrm{Kh}$ & $\mathrm{ml}$ & $\mathrm{W}$ & Sayedur563 \\
\hline *'Ocimum americanum L. & Ban tulshi & NMS & Herb, e & $\mathrm{Oz}, \mathrm{Pz} ; \mathrm{Sr}, \mathrm{Kh}$ & $\mathrm{ml}$, os & M & Sayedur1538 \\
\hline "O. sanctum L. & Tulshi & NMS & Herb, e & $\mathrm{Oz}, \mathrm{Pz} ; \mathrm{Sr}, \mathrm{Kh}$ & $\mathrm{ml}$, os & M & Sayedur514 \\
\hline \multicolumn{8}{|l|}{ Plantaginaceae Juss. } \\
\hline${ }^{*}$ Limnophila aromatica (Lam.) Merr. & Pani karpur & NMS & Herb, a & $\mathrm{Oz}, \mathrm{Mz} ; \mathrm{Sr}$ & wt & Aw & Sayedur694 \\
\hline $\begin{array}{l}\text { Mecardonia procumbens (Mill.) } \\
\text { Small. }\end{array}$ & Mikardan & NMS & Herb, $p$ & $\mathrm{Az} ; \mathrm{Ar}$ & $\mathrm{ml}$ & W & Sayedur1010 \\
\hline \multicolumn{8}{|l|}{ Scrophulariaceae Juss. } \\
\hline Adenosma indianum (Lour.) Mirr. & Borokesuti & NMS & Herb, e & $\mathrm{Mz} ; \mathrm{Sr}$ & $\mathrm{ml}$ & W & N1198 \\
\hline${ }^{*}$ Bacopa monnieri (L.) Pennell & Brammi & NMS & Herb, $p$ & $\mathrm{Mz}, \mathrm{Pz} ; \mathrm{Sr}, \mathrm{Kh}$ & $\mathrm{ml}, \mathrm{wt}$ & M & Sayedur1161 \\
\hline $\begin{array}{l}\text { Centranthera tranquebarica } \\
\text { (Spreng.) Merr. }\end{array}$ & Pashmicentra & NMS & Herb, $p$ & $\mathrm{Mz}-\mathrm{Pz} ; \mathrm{Sr}, \mathrm{Kh}$ & $\mathrm{gl}$ & $\mathrm{W}$ & Sayedur689 \\
\hline $\begin{array}{l}\text { Lindernia anagallis (Burm. f.) } \\
\text { Pennell. }\end{array}$ & Panighas & NMS & Herb, $p$ & $\mathrm{Oz}-\mathrm{Mz} ; \mathrm{Sr}, \mathrm{Kh}$ & $\mathrm{ml}, \mathrm{gl}$ & $\mathrm{W}$ & Sayedur503 \\
\hline L. antipoda (L.) Alston, & Sada panighas & NMS & Herb, $p$ & $\mathrm{Oz}, \mathrm{Mz} ; \mathrm{Sr}, \mathrm{Kh}$ & $\mathrm{ml}$ & $\mathrm{W}$ & Sayedur1226 \\
\hline L. ciliata (Colsm.) Pennell & Bhuijui & NMS & Herb, $p$ & $\mathrm{Oz}, \mathrm{Pz} ; \mathrm{Sr}, \mathrm{Kh}$ & $\mathrm{ml}, \mathrm{gl}$ & $\mathrm{W}$ & Sayedur231 \\
\hline${ }^{*}$ L. crustacea (L.) F. Muell. & Chapraghas & NMS & Herb, $p$ & $\mathrm{Oz}, \mathrm{Pz} ; \mathrm{Sr}, \mathrm{Kh}$ & $\mathrm{ml}, \mathrm{gl}$ & $\mathrm{W}$ & Sayedur585 \\
\hline L. procumbens (Krock.) Borbás & Bakpuspa & NMS & Herb, $p$ & $\mathrm{Oz}, \mathrm{Pz} ; \mathrm{Sr}, \mathrm{Kh}$ & $\mathrm{ml}, \mathrm{gl}$ & $\mathrm{W}$ & Mosharof136 \\
\hline L. pusilla (Willd.) Bold. & Pusichapra & NMS & Herb, $p$ & $\mathrm{Oz}, \mathrm{Pz} ; \mathrm{Sr}, \mathrm{Kh}$ & Ml & $\mathrm{W}$ & Sayedur498 \\
\hline "Scoparia dulcis L. & Misridana & NMS & Herb, e & $\mathrm{Oz}, \mathrm{Pz} ; \mathrm{Sr}, \mathrm{Kh}$ & $\mathrm{ml}, \mathrm{gl}$ & $\mathrm{W}$ & Sayedur777 \\
\hline
\end{tabular}


Table 2. Contd.

\begin{tabular}{|c|c|c|c|c|c|c|c|}
\hline Scientific name & Bangla name & $\mathrm{MC}$ & Habit & Distribution & Habitat & Use & RS \\
\hline \multicolumn{8}{|l|}{ Acanthaceae Juss. } \\
\hline${ }^{*}$ Acanthus ilicifolius L. & Hargoza & MS & Shrub & $\mathrm{Az} ; \mathrm{Ar}$ & $\mathrm{fm}, \mathrm{rb}$ & $\mathrm{M}, \mathrm{W}$ & Sayedur832 \\
\hline *A. volubilis Wall. & Lata hargoza & MAS & Herb, c & $\mathrm{Mz} ; \mathrm{Kh}$ & $\mathrm{fm}, \mathrm{rb}$ & $\mathrm{W}$ & Sayedur366 \\
\hline $\begin{array}{l}{ }^{*} \text { Hemigraphis hirta (Vahl) T. } \\
\text { Anderson }\end{array}$ & Buripana & NMS & Herb, $p$ & $\mathrm{Oz} ; \mathrm{Sr}$ & $\mathrm{ml}$ & $\mathrm{W}$ & Sayedur886 \\
\hline${ }^{*}$ Hygrophila erecta (Burm. f.) Hochr. & Filareck & NMS & Herb, e & $\mathrm{Mz}, \mathrm{Pz} ; \mathrm{Kh}, \mathrm{St}$ & wt & $\mathrm{W}$ & Sayedur1155 \\
\hline H. polysperma (Roxb.) T. Anderson & Alai kali & NMS & Herb, $p$ & $\mathrm{Mz}, \mathrm{Pz} ; \mathrm{Kh}, \mathrm{St}$ & wt & $\mathrm{W}$ & Sayedur727 \\
\hline${ }^{*} H$. quadrivalvis (Buch.-Ham.) Nees. & Unknown & NMS & Herb, $p$ & $\mathrm{Mz}, \mathrm{Pz} ; \mathrm{Kh}, \mathrm{St}$ & wt & $\mathrm{W}$ & Sayedur15 \\
\hline Justicia adhatoda L. & Bashak & NMS & Shrub & $\mathrm{Oz} ; \mathrm{Sr}$ & $\mathrm{ml}$ & M & Mosharof123 \\
\hline J. diffusa Willd. & Pitapapra & NMS & Herb, $p$ & $\mathrm{Oz} ; \mathrm{Sr}$ & $\mathrm{ml}$ & $\mathrm{W}$ & Mosharof124 \\
\hline $\begin{array}{l}\text { Nelsonia canescens (Lam.) Spreng. } \\
\text { Bignoniaceae Juss. }\end{array}$ & Paramul & NMS & Herb, $p$ & $\mathrm{Oz}, \mathrm{Mz} ; \mathrm{Sr}$ & $\mathrm{ml}$ & $\mathrm{W}$ & Sayedur391 \\
\hline $\begin{array}{l}\text { "Dolichandrone spathacea (L.f.) } \\
\text { Seem. }\end{array}$ & Garshingia & MAS & Tree, $\mathrm{s}$ & $\mathrm{Oz}, \mathrm{Mz} ; \mathrm{Sr}$ & $\mathrm{fm}, \mathrm{rb}$ & M & Sayedur1265 \\
\hline $\begin{array}{l}\text { Pajanelia longifolia K. Schum. } \\
\text { Campanulaceae Juss. }\end{array}$ & Pajanelia & NMS & Tree, $\mathrm{m}$ & $\mathrm{Oz} ; \mathrm{Sr}$ & $\mathrm{fm}, \mathrm{rb}$ & M & Sayedur926 \\
\hline $\begin{array}{l}\text { Sphenoclea zeylanica Gaertn. } \\
\text { Rubiaceae Juss. }\end{array}$ & Unknown & NMS & Herb, e & $\mathrm{Oz}, \mathrm{Mz} ; \mathrm{Sr}, \mathrm{Ch}$ & wt & $\mathrm{W}$ & Sayedur1312 \\
\hline $\begin{array}{l}\text { Dentella repens (L.) J.R. Forst. \& G. } \\
\text { Forst. }\end{array}$ & Bhuipat & NMS & Herb, $p$ & $\mathrm{Az} ; \mathrm{Sr}, \mathrm{Kh}$ & $\mathrm{gl}, \mathrm{ml}$ & $\mathrm{W}$ & Sayedur1235 \\
\hline D. serpyllifolia Wall. ex Craib & Bhuipat & NMS & Herb, $p$ & $\mathrm{Az} ; \mathrm{Sr}, \mathrm{Kh}$ & $\mathrm{gl}, \mathrm{ml}$ & $\mathrm{W}$ & Sayedur889 \\
\hline Gardenia jasminoides J. Ellis & Gondhoraj & NMS & Shrub & $\mathrm{Mz} ; \mathrm{Ch}$ & $\mathrm{ml}(\mathrm{pl})$ & $\mathrm{O}$ & Sayedur1133 \\
\hline Hedyotis biflora (L.) Lam. & Unknown & NMS & Herb, $p$ & $\mathrm{Az} ; \mathrm{Sr}, \mathrm{Kh}$ & $\mathrm{gl}, \mathrm{ml}$ & $\mathrm{W}$ & Sayedur1054 \\
\hline${ }^{*} H$. diffusa Willd. & Panki & NMS & Herb, $p$ & $\mathrm{Az} ; \mathrm{Ar}$ & $\mathrm{ml}$ & $\mathrm{M}, \mathrm{W}$ & Sayedur445 \\
\hline $\begin{array}{l}\text { "Hypobathrum racemosum (Roxb.) } \\
\text { Kurz }\end{array}$ & Peetunga & NMS & Tree, $\mathrm{s}$ & $\mathrm{Oz}, \mathrm{Mz} ; \mathrm{Sr}$ & $\mathrm{fm}, \mathrm{rb}$ & $\mathrm{W}, \mathrm{Fw}$ & Sayedur310 \\
\hline Ixora pavetta Andrews & Swet rangon & NMS & Shrub & $\mathrm{Az} ; \mathrm{Sr}, \mathrm{Kh}, \mathrm{St}$ & fm, os & $\mathrm{W}, \mathrm{O}$ & Sayedur465 \\
\hline "Morinda citrifolia $\mathrm{L}$. & Borochand & MAS & Shrub & $\mathrm{Oz} ; \mathrm{Sr}$ & $\mathrm{fm}, \mathrm{rb}$ & $\mathrm{W}$ & sayedur1268 \\
\hline $\begin{array}{l}\text { Neolamarckia cadamba (Roxb.) } \\
\text { Bosser }\end{array}$ & Kadam & NMS & Tree, 1 & $\mathrm{Oz} ; \mathrm{Sr}$ & $\mathrm{ml}$ & $\mathrm{T}, \mathrm{Fw}$ & Sayedur1330 \\
\hline $\begin{array}{l}\text { Oldenlandia corymbosa L. } \\
\text { Asteraceae Bercht. \& J. Presl }\end{array}$ & Khetpapra & NMS & Herb, $p$ & $\mathrm{Oz}, \mathrm{Pz} ; \mathrm{Sr}, \mathrm{Kh}$ & $\mathrm{ml}, \mathrm{gl}$ & $\mathrm{W}$ & Sayedur232 \\
\hline Acmella calva (DC.) R.K. Jansen & Surja konnya & NMS & Herb, $p$ & $\mathrm{Az} ; \mathrm{Ar}$ & $\mathrm{ml}$ & $\mathrm{M}, \mathrm{W}$ & Sayedur334 \\
\hline *Ageratum conyzoides L. & Fulkuri & NMS & Herb, e & $\mathrm{Oz}, \mathrm{Pz} ; \mathrm{Sr}, \mathrm{Kh}$ & $\mathrm{ml}$ & $\mathrm{M}, \mathrm{W}$ & Sayedur355 \\
\hline *Blumea bifoliata (L.) DC. & Unknown & NMS & Herb, $p$ & $\mathrm{Mz}, \mathrm{Pz} ; \mathrm{Kh}, \mathrm{St}$ & $\mathrm{gl}, \mathrm{ml}$ & $\mathrm{W}$ & Sayedur369 \\
\hline B. lacera (Burm. f.) DC. & Shialmutra & NMS & Herb, e & $\mathrm{Az} ; \mathrm{Ar}$ & $\mathrm{ml}$ & $\mathrm{M}, \mathrm{W}$ & Sayedur394 \\
\hline B. membranacea DC. & Unknown & NMS & Herb, e & $\mathrm{Mz} ; \mathrm{Sr}$ & $\mathrm{gl}$ & $\mathrm{W}$ & Sayedur610 \\
\hline B. oxyodonta DC. & Katapata & NMS & Herb, e & $\mathrm{Oz}, \mathrm{Mz} ; \mathrm{Sr}$ & $\mathrm{ml}$ & $\mathrm{W}$ & Sayedur863 \\
\hline $\begin{array}{l}\text { Chromolaena odorata (L.) R.M. } \\
\text { King \& H. Rob. }\end{array}$ & Boroshialmuti & NMS & Herb, e & $\mathrm{Oz}, \mathrm{Pz} ; \mathrm{Sr}, \mathrm{Kh}$ & $\mathrm{ml}$ & $\mathrm{W}$ & Sayedur368 \\
\hline *Conyza semipinnatifida Wall. ex DC & Coniza & NMS & Herb, $p$ & $\mathrm{Mz}, \mathrm{Pz} ; \mathrm{Kh}, \mathrm{St}$ & $\mathrm{gl}$ & $\mathrm{W}$ & Sayedur568 \\
\hline Eclipta alba (L.) Hassk. & Kalokeshi & NMS & Herb, $p$ & $\mathrm{Oz}, \mathrm{Pz} ; \mathrm{Sr}, \mathrm{Kh}$ & $\mathrm{ml}, \mathrm{gl}$ & $\mathrm{W}$ & Sayedur1294 \\
\hline
\end{tabular}


Table 2. Contd.

\begin{tabular}{|c|c|c|c|c|c|c|c|}
\hline Scientific name & Bangla name & $\mathrm{MC}$ & Habit & Distribution & Habitat & Use & RS \\
\hline Elephantopus scaber L. & Hostipado & NMS & Herb, e & $\mathrm{Oz} ; \mathrm{Sr}$ & $\mathrm{ml}$ & $\mathrm{W}$ & Mosharof89 \\
\hline Emilia sonchifolia (L.) DC. & Mechitra & NMS & Herb, $p$ & $\mathrm{Oz} ; \mathrm{Sr}$ & $\mathrm{ml}$ & W & Mosharof91 \\
\hline Enhydra fluctuans Lour. & Helencha & NMS & Herb, a & $\mathrm{Oz}, \mathrm{Mz} ; \mathrm{Sr}, \mathrm{Ch}$ & $\mathrm{mt}$ & $\mathrm{V}, \mathrm{Aw}$ & Sayedur378 \\
\hline $\begin{array}{l}\text { *Grangea maderaspatana (L.) } \\
\text { Poir. }\end{array}$ & Namuti & NMS & Herb, $p$ & $\mathrm{Oz} ; \mathrm{Sr}$ & $\mathrm{ml}$ & $\mathrm{W}$ & Sayedur 1320 \\
\hline Launaea asplenifolia Hook. f. & Tikdana & NMS & Herb, $p$ & $\mathrm{Pz} ; \mathrm{Kh}, \mathrm{St}$ & gl & W & Sayedur581 \\
\hline${ }^{*}$ L. sarmentosa (Willd.) Kuntze & Menthosdana & NMS & Herb, $p$ & $\mathrm{Pz} ; \mathrm{Kh}$ & gl & W & Sayedur483 \\
\hline $\begin{array}{l}\text { Mikania cordata (Burm.f.) } \\
\text { B.L. Rob. }\end{array}$ & Assam lata & NMS & Herb, c & $\mathrm{Oz}, \mathrm{Pz} ; \mathrm{Sr}, \mathrm{Kh}$ & $\mathrm{fm}, \mathrm{ml}$ & $\mathrm{M}, \mathrm{W}$ & Sayedur1298 \\
\hline $\begin{array}{l}\text { Pseudognaphalium luteoalbum } \\
\text { (L.) Hilliard \& B.L. Burtt }\end{array}$ & Barakamra & NMS & Herb, e & $\mathrm{Oz}, \mathrm{Mz} ; \mathrm{Sr}$ & $\mathrm{ml}$ & W & Sayedur725 \\
\hline Sonchus oleraceus L. & Unknown & NMS & Herb, e & $\mathrm{Oz} ; \mathrm{Sr}$ & $\mathrm{ml}, \mathrm{gl}$ & W & Sayedur 2128 \\
\hline S. wightianus DC. & Unknown & NMS & Herb, e & $\mathrm{Oz} ; \mathrm{Sr}$ & $\mathrm{ml}, \mathrm{gl}$ & W & Sayedur 288 \\
\hline "Sphaeranthus africanus L. & Gangasag & NMS & Herb, $p$ & $\mathrm{Oz} ; \mathrm{Sr}$ & $\mathrm{ml}$ & W & Sayedur 1325 \\
\hline S. indicus L. & Mundi & NMS & Herb, $p$ & $\mathrm{Oz} ; \mathrm{Sr}$ & $\mathrm{ml}$ & $\mathrm{W}$ & Sayedur 720 \\
\hline $\begin{array}{l}{ }^{*} \text { Sphagneticola calendulacea } \\
\text { (L.) Pruski }\end{array}$ & Bhimraj & NMS & Herb, $p$ & $\mathrm{Oz} ; \mathrm{Sr}$ & $\mathrm{ml}$ & $\mathrm{O}$ & Sayedur2173 \\
\hline Tridax procumbens L. & Tridhara & NMS & Herb, $p$ & $\mathrm{Oz}, \mathrm{Pz} ; \mathrm{Sr}, \mathrm{Kh}$ & $\mathrm{ml}, \mathrm{gl}$ & W & Mosharof212 \\
\hline "Vernonia cinerea (L.) Less. & Kuksim & NMS & Herb, e & $\mathrm{Az} ; \mathrm{Ar}$ & $\mathrm{ml}, \mathrm{gl}$ & W & Sayedur1100 \\
\hline V. divergens (DC.) Edgew. & Bichutivernon & NMS & Herb, e & $\mathrm{Mz} ; \mathrm{Sr}, \mathrm{Kh}$ & $\mathrm{ml}$, os & W & Sayedur64 \\
\hline "Wollastonia biflora (L.) DC. & Wedella & MAS & Herb, $p$ & $\mathrm{Oz} ; \mathrm{Sr}$ & $\mathrm{ml}$ & M & Sayedur996 \\
\hline $\begin{array}{l}\text { Xanthium indicum J. Koenig ex } \\
\text { Roxb. }\end{array}$ & Ghagra & NMS & Herb, e & $\mathrm{Oz} ; \mathrm{Sr}, \mathrm{Ch}$ & $\mathrm{ml}$ & $\mathrm{M}, \mathrm{W}$ & Mosharof239 \\
\hline \multicolumn{8}{|c|}{ LILIOPSIDA (MONOCOTYLEDONS) } \\
\hline \multicolumn{8}{|c|}{ Alismataceae Vent. } \\
\hline \multicolumn{7}{|l|}{ Hydrocharitaceae Juss. } & Sayedur171 \\
\hline $\begin{array}{l}{ }^{*} \text { Hydrilla verticillata (L. f.) } \\
\text { Royle }\end{array}$ & Jhangi & NMS & Herb, a & $\mathrm{Oz}, \mathrm{Mz} ; \mathrm{Sr}, \mathrm{Ch}$ & wt & $\mathrm{Ff}$ & Sayedur1295 \\
\hline $\begin{array}{l}\text { *Vallisneria spiralis L. } \\
\text { Aponogetonaceae Planch. }\end{array}$ & Pataseola & NMS & Herb, a & $\mathrm{Az} ; \mathrm{Ar}$ & wt & Aw & Sayedur759 \\
\hline $\begin{array}{l}\text { Aponogeton echinatus Roxb. } \\
\text { Najadaceae Juss. }\end{array}$ & Ghecu & NMS & Herb, a & $\mathrm{Oz} ; \mathrm{Ch}$ & wt & $\mathrm{V}$ & Sayedur1195 \\
\hline "Najas minor All. & Boro jhaji & MAS & Herb, a & $\mathrm{Pz} ; \mathrm{St}$ & wt & $\mathrm{Ff}$ & Sayedur1929 \\
\hline \multicolumn{8}{|l|}{ Arecaceae Bercht. \& J. Presl } \\
\hline${ }^{*}$ Areca catechu $\mathrm{L}$ & Supari & NMS & Tree, 1 & $\mathrm{Az} ; \mathrm{Ar}$ & $\mathrm{ml}(\mathrm{pl})$ & M & Sayedur2194 \\
\hline Borassus flabellifer L. & Tal & NMS & Tree, 1 & $\mathrm{Az} ; \mathrm{Ar}$ & $\mathrm{ml}(\mathrm{pl})$ & $\mathrm{Fr}, \mathrm{Du}$ & Sayedur163 \\
\hline "Calamus tenuis Roxb. & Sachi bet & NMS & Herb, c & $\mathrm{Oz}, \mathrm{Mz} ; \mathrm{Sr}$ & wd, fm & $\mathrm{Fn}, \mathrm{Du}$ & Sayedur307 \\
\hline *Cocos nucifera $\mathrm{L}$. & Narikel & NMS & Tree, 1 & $\mathrm{Az} ; \mathrm{Ar}$ & $\mathrm{ml}(\mathrm{pl})$ & $\mathrm{Fr}, \mathrm{Du}$ & Sayedur41 \\
\hline${ }^{*}$ Nypa fruticans Wurmb & Golpata & MS & Tree, $\mathrm{s}$ & $\mathrm{Az} ; \mathrm{Ar}$ & wd & $\mathrm{Fr}, \mathrm{Du}$ & Sayedur423 \\
\hline * Phoenix paludosa Roxb. & Hental & MS & Tree, $\mathrm{s}$ & $\mathrm{Az} ; \mathrm{Ar}$ & wd & $\mathrm{Du}$ & Sayedur712 \\
\hline P. sylvestris (L.) Roxb. & Khejur & NMS & Tree, 1 & $\mathrm{Az} ; \mathrm{Ar}$ & $\mathrm{ml}$ & $\mathrm{Ju}, \mathrm{Du}$ & Sayedur392 \\
\hline
\end{tabular}


Table 2. Contd.

\begin{tabular}{|c|c|c|c|c|c|c|c|}
\hline Scientific name & Bangla name & $\mathrm{MC}$ & Habit & Distribution & Habitat & Use & $\mathrm{RS}$ \\
\hline \multicolumn{8}{|l|}{ Pandanaceae R. Br. } \\
\hline *Pandanus foetidus Roxb. & Keya kanta & NMS & Shrub & $\mathrm{Az} ; \mathrm{Sr}, \mathrm{Ch}, \mathrm{St}$ & $\mathrm{rb}, \mathrm{fm}$ & $\mathrm{M}, \mathrm{W}$ & Sayedur229 \\
\hline${ }^{*}$ P. tectorius Parkinson & Keya & MAS & Tree, s & $\mathrm{Mz}, \mathrm{Pz} ; \mathrm{Sr}, \mathrm{St}$ & $\mathrm{fm}$ & $\mathrm{W}$ & Sayedur499 \\
\hline \multicolumn{8}{|l|}{ Araceae Juss. } \\
\hline Alocasia macrorrhizos (L.) G. Don & Mankachu & NMS & Herb, e & $\mathrm{Oz}, \mathrm{Mz} ; \mathrm{Sr}, \mathrm{Ch}$ & $\mathrm{ml}$ & $\mathrm{V}, \mathrm{M}$ & Sayedur1333 \\
\hline Colocasia esculenta (L.) Schott & Kochu & MAS & Herb, e & $\mathrm{Oz}, \mathrm{Pz} ; \mathrm{Sr}, \mathrm{Kh}$ & $\mathrm{ml}, \mathrm{wt}$ & $\mathrm{V}, \mathrm{M}$ & Sayedur358 \\
\hline $\begin{array}{l}{ }^{*} \text { Cryptocoryne ciliata (Roxb.) } \\
\text { Fisch. ex Wydl. }\end{array}$ & Kerali & MAS & Herb, e & $\mathrm{Oz}, \mathrm{Mz} ; \mathrm{Sr}, \mathrm{Kh}$ & wt & Aw & Sayedur369 \\
\hline Lasia spinosa (L.) Thwaites & Kanta kachu & MAS & Herb, e & $\mathrm{Oz} ; \mathrm{Sr}$ & wt & $\mathrm{V}, \mathrm{M}$ & Sayedur312 \\
\hline "Pistia stratiotes L. & Topapana & NMS & Herb, a & $\mathrm{Oz} ; \mathrm{Sr}$ & wt & M & Sayedur652 \\
\hline Typhonium trilobatum (L.) Schott. & Ghetkachu & NMS & Herb, e & $\mathrm{Oz}, \mathrm{Pz} ; \mathrm{Sr}, \mathrm{Kh}$ & $\mathrm{ml}$ & $\mathrm{V}$ & Sayedur1318 \\
\hline \multicolumn{8}{|c|}{ Lemnaceae Martinov } \\
\hline Lemna perpusilla Torr. & Khudipana & NMS & Herb, a & $\mathrm{Pz} ; \mathrm{St}$ & wt & $\mathrm{Ff}, \mathrm{Mn}$ & Sayedur1104 \\
\hline \multicolumn{8}{|l|}{ Commelinaceae Mirb. } \\
\hline *Commelina benghalensis L. & Kanchira & NMS & Herb, $p$ & $\mathrm{Az} ; \mathrm{Ar}$ & $\mathrm{gl}, \mathrm{ml}$ & M & Sayedur63 \\
\hline C. longifolia Lam. & Pani kanchira & NMS & Herb, $p$ & $\mathrm{Az} ; \mathrm{Ar}$ & $\mathrm{gl}, \mathrm{ml}$ & $\mathrm{W}$ & Sayedur1307 \\
\hline Murdannia blumei (Hassk.) Brenan & Nil murdan & NMS & Herb, $p$ & $\mathrm{Mz} ; \mathrm{Sr}$ & $\mathrm{gl}, \mathrm{ml}$ & $\mathrm{W}$ & Sayedur206 \\
\hline $\begin{array}{l}\text { M. loriformis (Hassk.) R.S. Rao \& } \\
\text { Kammathy }\end{array}$ & Lori murdan & NMS & Herb, $p$ & $\mathrm{Pz} ; \mathrm{Kh}$ & $\mathrm{gl}, \mathrm{ml}$ & $\mathrm{W}$ & Sayedur164 \\
\hline${ }^{*}$ M. nudiflora (L.) Brenan & Kanduli & NMS & Herb, $p$ & $\mathrm{Mz} ; \mathrm{Sr}$ & $\mathrm{gl}, \mathrm{ml}$ & $\mathrm{W}$ & Sayedur141 \\
\hline M. vaginata (L.) G. Brückn. & Dhaka murdan & NMS & Herb, $p$ & $\mathrm{Pz} ; \mathrm{Kh}$ & $\mathrm{gl}, \mathrm{ml}$ & $\mathrm{W}$ & Sayedur108 \\
\hline \multicolumn{8}{|l|}{ Flagellariaceae Dumort. } \\
\hline "Flagellaria indica L. & Abeti & MAS & Herb, c & $\mathrm{Oz}, \mathrm{Mz} ; \mathrm{Sr}$ & $\mathrm{fm}, \mathrm{rb}$ & M & Sayedur200 \\
\hline \multicolumn{8}{|l|}{ Cyperaceae Juss. } \\
\hline $\begin{array}{l}\text { Bulbostylis barbata (Rottb.) C.B. } \\
\text { Clarke }\end{array}$ & Bulbobata & NMS & Herb, e & $\mathrm{Mz} ; \mathrm{Sr}$ & $\mathrm{gl}$ & $\mathrm{Sb}$ & Sayedur182 \\
\hline Cyperus articulatus L. & Joraghasi & NMS & Herb, e & $\mathrm{Mz}, \mathrm{Pz} ; \mathrm{Sr}, \mathrm{Kh}$ & wt & $\mathrm{Du}$ & Sayedur227 \\
\hline C. compressus L. & Chanch & NMS & Herb, e & $\mathrm{Mz} ; \mathrm{Sr}$ & $\mathrm{gl}$ & $\mathrm{W}$ & Sayedur165 \\
\hline C. cuspidatus Kunth & Sagormukhi & NMS & Herb, e & $\mathrm{Mz}, \mathrm{Pz} ; \mathrm{Sr}, \mathrm{Kh}$ & $\mathrm{gl}$ & $\mathrm{W}$ & Sayedur166 \\
\hline C. difformis $\mathrm{L}$. & Behua ghasi & NMS & Herb, e & $\mathrm{Mz}, \mathrm{Pz} ; \mathrm{Sr}, \mathrm{Kh}$ & gl & $\mathrm{W}$ & $N 1215$ \\
\hline C. digitatus Roxb. & Hath ghasi & NMS & Herb, e & $\mathrm{Oz}, \mathrm{Mz} ; \mathrm{Sr}$ & wt & Aw & Sayedur268 \\
\hline${ }^{*}$ C. exaltatus Retz. & Tata ghasi & NMS & Herb, e & $\mathrm{Oz} ; \mathrm{Sr}$ & wt & Mt & Sayedur 270 \\
\hline C. involucratus Rottb. & Sata ghasi & NMS & Herb, e & $\mathrm{Pz} ; \mathrm{Kh}$ & $\mathrm{gl}$ & $\mathrm{O}$ & Sayedur1153 \\
\hline C. iria L. & Barachucha & NMS & Herb, e & $\mathrm{Pz} ; \mathrm{St}$ & wt & $\mathrm{Fd}, \mathrm{M}$ & Sayedur42 \\
\hline${ }^{*}$ C. javanicus Houtt. & Java ghasi & MAS & Herb, e & $\mathrm{Az} ; \mathrm{Sr}, \mathrm{Kh}$ & gl & $\mathrm{Sb}$ & Sayedur214 \\
\hline${ }^{*}$ C. malaccensis Lam. & Shumati pati & MAS & Herb, e & $\mathrm{Az} ; \mathrm{Ar}$ & wt & $\mathrm{Du}$ & Sayedur191 \\
\hline C. rotundus L. & Nagarmutha & NMS & Herb, e & $\mathrm{Oz}, \mathrm{Pz} ; \mathrm{Sr}, \mathrm{Kh}$ & $\mathrm{gl}$ & $\mathrm{M}, \mathrm{W}$ & Sayedur167 \\
\hline C. tenuiculmis Boeckeler & Tonimutha & NMS & Herb, e & $\mathrm{Pz} ; \mathrm{St}$ & wt & AW & Sayedur 43 \\
\hline $\begin{array}{l}\text { Eleocharis dulcis (Burm. f.) Trin. } \\
\text { ex Hensch. }\end{array}$ & Mishti ghasi & MAS & Herb, e & $\mathrm{Pz} ; \mathrm{St}$ & wt & $\mathrm{V}$ & Sayedur1503 \\
\hline E. geniculata (L.) Roem. \& Schult. & Jora ghasi & NMS & Herb, e & $\mathrm{Oz}, \mathrm{Pz} ; \mathrm{Sr}, \mathrm{Kh}$ & gl & $\mathrm{W}$ & Sayedur183 \\
\hline $\begin{array}{l}{ }^{*} \text { E. spiralis (Rottb.) Roem. \& } \\
\text { Schult. }\end{array}$ & Ghurni ghasi & MAS & Herb, e & $\mathrm{Pz} ; \mathrm{Kh}$ & wt & Aw & Sayedur562 \\
\hline
\end{tabular}


Table 2. Contd.

\begin{tabular}{|c|c|c|c|c|c|c|c|}
\hline Scientific name & Bangla name & $\mathrm{MC}$ & Habit & Distribution & Habitat & Use & $\mathrm{RS}$ \\
\hline Fimbristylis acuminata Vahl & Chosa fimbry & NMS & Herb, e & $\mathrm{Mz}, \mathrm{Pz} ; \mathrm{Sr}, \mathrm{Kh}$ & gl & $\mathrm{Sb}$ & Sayedur683 \\
\hline F. autumnalis (L.) Roem. \& Schult. & Fimbry & NMS & Herb, e & $\mathrm{Mz} ; \mathrm{Sr}$ & gl & $\mathrm{Sb}$ & Sayedur1277 \\
\hline F. cymosa R. Br. & Mosa fimbry & MAS & Herb, e & $\mathrm{Oz}, \mathrm{Pz} ; \mathrm{Sr}, \mathrm{Kh}$ & $\mathrm{gl}$ & W & Sayedur622 \\
\hline F. dichotoma (L.) Vahl & Bara nirbishi & NMS & Herb, e & $\mathrm{Az} ; \mathrm{Ar}$ & $\mathrm{gl}$ & $\mathrm{W}$ & Sayedur487 \\
\hline F. disticha Boeckeler & Tika fimbry & NMS & Herb, e & $\mathrm{Oz}, \mathrm{Pz} ; \mathrm{Sr}, \mathrm{Kh}$ & $\mathrm{gl}$ & $\mathrm{W}$ & Sayedur199 \\
\hline${ }^{*}$ F. ferruginea (L.) Vahl & Gini fimbry & MAS & Herb, e & $\mathrm{Az} ; \mathrm{Ar}$ & gl, wt & $\mathrm{W}, \mathrm{Sb}$ & Sayedur837 \\
\hline F. littoralis Gaudich. & Litto fimbry & NMS & Herb, e & $\mathrm{Mz}, \mathrm{Pz} ; \mathrm{Sr}, \mathrm{Kh}$ & $\mathrm{gl}$ & $\mathrm{Sb}$ & N1234 \\
\hline${ }^{*}$ F. ovata (Burm. f.) J. Kern & Marmari & NMS & Herb, e & $\mathrm{Mz}, \mathrm{Pz} ; \mathrm{Sr}, \mathrm{Kh}$ & $\mathrm{gl}$ & W & Sayedur168 \\
\hline F. quinquangularis (Vahl) Kunth & Pachkona fibmry & NMS & Herb, e & $\mathrm{Pz} ; \mathrm{Kh}$ & gl & W & Sayedur126 \\
\hline F. bisumbellata (Forssk.) Bubani & Dula fimbry & NMS & Herb, e & $\mathrm{Pz} ; \mathrm{Kh}$ & gl & $\mathrm{W}$ & Sayedur169 \\
\hline F. squarrosa Vahl & Zumka chech & NMS & Herb, e & $\mathrm{Mz} ; \mathrm{Sr}$ & $\mathrm{gl}$ & W & Sayedur194 \\
\hline F. tetragona $\mathrm{R}$. Br. & Tetra fimbry & NMS & Herb, e & $\mathrm{Pz} ; \mathrm{Kh}$ & $\mathrm{gl}$ & $\mathrm{W}$ & Sayedur86 \\
\hline F. tristachya R. Br. & Trista fimbry & NMS & Herb, e & $\mathrm{Mz}, \mathrm{Pz} ; \mathrm{Sr}, \mathrm{Kh}$ & gl & $\mathrm{W}$ & Sayedur650 \\
\hline Fuirena ciliaris (L.) Roxb. & Poshmi ghasi & NMS & Herb, e & $\mathrm{Mz}, \mathrm{Pz} ; \mathrm{Sr}, \mathrm{Kh}$ & wt & AW & Sayedur186 \\
\hline F. umbellata Rottb. & Chati ghasi & NMS & Herb, e & $\mathrm{Mz}, \mathrm{Pz} ; \mathrm{Sr}, \mathrm{Kh}$ & wt & AW & Sayedur682 \\
\hline Kyllinga brevifolia Rottb. & Shabujnirbish & NMS & Herb, e & $\mathrm{Mz}, \mathrm{Pz} ; \mathrm{Sr}, \mathrm{Kh}$ & gl & $\mathrm{Fd}$ & Sayedur204 \\
\hline $\begin{array}{l}\text { K. nemoralis (J.R. Forst. \& G. } \\
\text { Forst.) Dandy ex Hutch. \& Dalziel }\end{array}$ & Subasinirbish & NMS & Herb, e & $\mathrm{Mz} ; \mathrm{Ch}$ & $\mathrm{ml}$ & $\mathrm{Fd}$ & Sayedur170 \\
\hline $\begin{array}{l}\text { "Pycreus polystachyos (Rottb.) P. } \\
\text { Beauv. }\end{array}$ & Pikppli ghasi & NMS & Herb, e & $\mathrm{Az} ; \mathrm{Ar}$ & $\mathrm{gl}, \mathrm{wt}$ & $\mathrm{W}, \mathrm{Sb}$ & Sayedur836 \\
\hline $\begin{array}{l}\text { P. sanguinolentus (Vahl) Nees ex } \\
\text { C.B. Clarke }\end{array}$ & Paikram ghasi & NMS & Herb, e & $\mathrm{Pz} ; \mathrm{Kh}$ & wt & AW & Sayedur85 \\
\hline P. uniloides (R. Br.) Urb. & Paikol ghasi & NMS & Herb, e & $\mathrm{Az} ; \mathrm{Ar}$ & $\mathrm{ml}, \mathrm{gl}$ & $\mathrm{W}$ & Sayedur3 \\
\hline Schoenoplectus articulatus (L.) Palla & Chechra & NMS & Herb, a & $\mathrm{Mz}, \mathrm{Pz} ; \mathrm{Sr}, \mathrm{Kh}$ & wt & $\mathrm{Fd}, \mathrm{M}$ & Sayedur679 \\
\hline *S. supinus (L.) Palla & Supipotpoti & NMS & Herb, a & $\mathrm{Oz} ; \mathrm{Ch}$ & $\mathrm{ml}$ & $\mathrm{W}$ & Sayedur1305 \\
\hline Scleria biflora Roxb. & Riaflora ghasi & NMS & Herb, e & $\mathrm{Mz} ; \mathrm{Sr}$ & $\mathrm{gl}$ & $\mathrm{Fd}$ & N1231 \\
\hline \multicolumn{8}{|l|}{ Poaceae Barnhart } \\
\hline Arundo donax L. & Baranal & NMS & Herb, e & $\mathrm{Mz} ; \mathrm{Ch}$ & wt & $\mathrm{Fd}, \mathrm{Du}$ & Sayedur935 \\
\hline $\begin{array}{l}\text { Axonopus compressus (Sw.) P. } \\
\text { Beauv. }\end{array}$ & Balla ghas & NMS & Herb, cr & $\mathrm{Az} ; \mathrm{Ar}$ & $\mathrm{gl}$ & $\mathrm{Sb}$ & Sayedur272 \\
\hline $\begin{array}{l}{ }^{*} \text { Bothriochloa bladhii (Retz.) S.T. } \\
\text { Blake }\end{array}$ & Gandha gourni & NMS & Herb, $p$ & $\mathrm{Mz} ; \mathrm{Sr}$ & $\mathrm{gl}$ & $\mathrm{Fd}$ & N1188 \\
\hline Brachiaria distachya (L.) Stapf & Cori ghas & NMS & Herb, $p$ & $\mathrm{Pz} ; \mathrm{Kh}$ & $\mathrm{ml}$ & $\mathrm{Fd}$ & Sayedur132 \\
\hline B. ramosa (L.) Stapf & Jhopa ghas & NMS & Herb, $p$ & $\mathrm{Oz} ; \mathrm{Sr}$ & $\mathrm{gl}$ & W & Sayedur320 \\
\hline $\begin{array}{l}{ }^{*} \text { Chrysopogon aciculatus (Retz.) } \\
\text { Trin. }\end{array}$ & Premkanta & NMS & Herb, e & $\mathrm{Mz}, \mathrm{Pz} ; \mathrm{Sr}, \mathrm{Kh}$ & $\mathrm{gl}$ & $\mathrm{W}$ & Sayedur90 \\
\hline${ }^{*}$ C. zizanioides (L.) Roberty & Bena & NMS & Herb, e & $\mathrm{Mz}, \mathrm{Pz} ; \mathrm{Sr}, \mathrm{Kh}$ & $\mathrm{gl}$ & $\mathrm{W}, \mathrm{Sb}$ & Sayedur218 \\
\hline Cynodon dactylon (L.) Pers. & Durba & MAS & Herb, cr & $\mathrm{Az} ; \mathrm{Ar}$ & $\mathrm{gl}$ & $\mathrm{M}, \mathrm{Sb}$ & Sayedur219 \\
\hline $\begin{array}{l}\text { *Dactyloctenium aegyptium (L.) } \\
\text { Willd. }\end{array}$ & Makra & NMS & Herb, e & $\mathrm{Mz}, \mathrm{Pz} ; \mathrm{Sr}, \mathrm{Kh}$ & $\mathrm{gl}$ & $\mathrm{Fd}, \mathrm{Sb}$ & Sayedur123 \\
\hline $\begin{array}{l}\text { Digitaria abludens (Roem. \& } \\
\text { Schult.) Veldkamp }\end{array}$ & Chirichira & NMS & Herb, $p$ & $\mathrm{Pz} ; \mathrm{Kh}$ & $\mathrm{gl}$ & $\mathrm{W}, \mathrm{Sb}$ & Sayedur1879 \\
\hline
\end{tabular}


Table 2. Contd.

\begin{tabular}{|c|c|c|c|c|c|c|c|}
\hline Scientific name & Bangla name & $\mathrm{MC}$ & Habit & Distribution & Habitat & Use & RS \\
\hline D. ciliaris (Retz.) Koeler & Kokjachira & NMS & Herb, $p$ & $\mathrm{Pz} ; \mathrm{Sr}, \mathrm{Kh}$ & $\mathrm{gl}$ & $\mathrm{W}, \mathrm{Sb}$ & Sayedur 2406 \\
\hline D. fuscescens (J. Presl) Henrard & Fusche ghas & NMS & Herb, $p$ & $\mathrm{Pz} ; \mathrm{Kh}$ & $\mathrm{gl}$ & $\mathrm{W}$ & Sayedur220 \\
\hline Diplacrum caricinum R. Br. & Plukram ghas & NMS & Herb, $p$ & $\mathrm{Mz}, \mathrm{Pz} ; \mathrm{Sr}, \mathrm{Kh}$ & $\mathrm{gl}$ & W & N1200 \\
\hline${ }^{*}$ Echinochloa colona (L.) Link & Shama ghas & NMS & Herb, e & $\mathrm{Az} ; \mathrm{Sr}, \mathrm{Kh}$ & wt & $\mathrm{Fd}$ & Sayedur278 \\
\hline${ }^{*}$ E. crus-galli (L.) P. Beauv. & Barashama ghas & NMS & Herb, a & $\mathrm{Az} ; \mathrm{Ar}$ & wt & $\mathrm{Fd}$ & Sayedur997 \\
\hline E. stagnina (Retz.) P. Beauv. & Parua ghas & NMS & Herb, a & $\mathrm{Mz}, \mathrm{Pz} ; \mathrm{Sr}, \mathrm{Kh}$ & wt & $\mathrm{Fd}$ & Sayedur998 \\
\hline${ }^{*}$ Eleusine indica (L.) Gaertn. & Malanga kuri & NMS & Herb, e & $\mathrm{Oz}, \mathrm{Pz} ; \mathrm{Sa}, \mathrm{St}$ & $\mathrm{ml}$ & $\mathrm{Sb}$ & Sayedur11 \\
\hline Eragrostis gangetica (Roxb.) Steud. & Chirakoni & NMS & Herb, $p$ & $\mathrm{Mz}, \mathrm{Pz} ; \mathrm{Sr}, \mathrm{Kh}$ & $\mathrm{gl}$ & $\mathrm{Sb}$ & N1230 \\
\hline $\begin{array}{l}\text { "E. tenella (L.) P. Beauv. ex Roem. } \\
\text { \& Schult }\end{array}$ & Koni ghas & NMS & Herb, $p$ & $\mathrm{Mz} ; \mathrm{Sr}$ & $\mathrm{gl}$ & $\mathrm{Sb}$ & N1274 \\
\hline E. unioloides (Retz.) Nees ex Steud. & Chira ghas & NMS & Herb, $p$ & $\mathrm{Mz} ; \mathrm{Sr}$ & $\mathrm{gl}$ & $\mathrm{Mn}, \mathrm{Sb}$ & N1208 \\
\hline $\begin{array}{l}\text { Eriochloa fatmensis (Hochst. \& } \\
\text { Steud.) Clayton }\end{array}$ & Motanol & NMS & Herb, $p$ & $\mathrm{Oz} ; \mathrm{Sr}$ & $\mathrm{gl}$ & W & Sayedur345 \\
\hline Hemarthria vaginata Buse & Chalia & NMS & Herb, $p$ & $\mathrm{Oz} ; \mathrm{Sr}$ & $\mathrm{gl}$ & $\mathrm{W}$ & Sayedur753 \\
\hline $\begin{array}{l}\text { *Imperata cylindrica (L.) Raeusch. } \\
\text { var. cylindrica }\end{array}$ & Chhon & NMS & Herb, e & $\mathrm{Az} ; \mathrm{Ar}$ & $\mathrm{gl}$ & $\mathrm{Sb}$ & Sayedur1542 \\
\hline "Leersia hexandra Sw. & Aralia ghas & NMS & Herb, $p$ & $\mathrm{Oz} ; \mathrm{Sr}$ & wt & $\mathrm{Fd}$ & Sayedur 2195 \\
\hline $\begin{array}{l}\text { "Myriostachya wightiana (Nees ex } \\
\text { Steud.) Hook. f. }\end{array}$ & Balya ghas & MAS & Herb, e & $\mathrm{Mz}, \mathrm{Pz} ; \mathrm{Ar}$ & $\mathrm{wt}, \mathrm{rb}$ & $\mathrm{Fd}$ & Sayedur45 \\
\hline $\begin{array}{l}\text { Oplismenus compositus (L.) P. } \\
\text { Beauv. }\end{array}$ & Gohur & NMS & Herb, $p$ & $\mathrm{Oz} ; \mathrm{Ch}$ & $\mathrm{ml}$ & $\mathrm{Fd}$ & Sayedur1306 \\
\hline Panicum cambogiense Balansa & Panicombo & NMS & Herb, e & $\mathrm{Mz} ; \mathrm{Sr}$ & $\mathrm{ml}$ & $\mathrm{Fd}$ & Sayedur324 \\
\hline P. maximum Jacq. & Gini ghas & MAS & Herb, e & $\mathrm{Oz} ; \mathrm{Sr}$ & wt & $\mathrm{Fd}$ & Sayedur115 \\
\hline${ }^{*} P$. paludosum Roxb. & Borali & NMS & Herb, e & $\mathrm{Mz}, \mathrm{Pz} ; \mathrm{Sr}, \mathrm{Kh}$ & wt & $\mathrm{Fd}$ & Sayedur84 \\
\hline${ }^{*}$ P. repens L. & Dhani ghas & NMS & Herb, e & $\mathrm{Az} ; \mathrm{Ar}$ & $\mathrm{gl}, \mathrm{ml}$ & $\mathrm{Fd}$ & Sayedur995 \\
\hline Paspalum conjugatum P.J. Bergius & Moishya ghas & NMS & Herb, e & $\mathrm{Oz}, \mathrm{Mz} ; \mathrm{Sr}$ & $\mathrm{gl}$ & $\mathrm{Fd}$ & Sayedur266 \\
\hline${ }^{*}$ P. distichum L. & Gitta ghas & NMS & Herb, $p$ & $\mathrm{Oz}, \mathrm{Mz} ; \mathrm{Sr}, \mathrm{Ch}$ & $\mathrm{gl}$ & $\mathrm{Sb}, \mathrm{Fd}$ & Sayedur285 \\
\hline${ }^{*}$ P. scrobiculatum L. & Khodadhan & NMS & Herb, e & $\mathrm{Mz} ; \mathrm{Sr}$ & wt & $\mathrm{Fd}$ & Sayedur195 \\
\hline P. vaginatum $\mathrm{Sw}$ & Gina ghas & MAS & Herb, $p$ & $\mathrm{Az} ; \mathrm{Ar}$ & $\mathrm{gl}$, wt & $\mathrm{Fd}, \mathrm{Sb}$ & Sayedur47 \\
\hline $\begin{array}{l}\text { "Phragmites karka (Retz.) Trin. ex } \\
\text { Steud. }\end{array}$ & Nal khagra & MAS & Herb, e & $\mathrm{Oz}, \mathrm{Mz} ; \mathrm{Sr}$ & wt & Fd, Du & Sayedur516 \\
\hline $\begin{array}{l}{ }^{*} \text { Porteresia coarctata (Roxb.) } \\
\text { Takeoka }\end{array}$ & Dhansi & NMS & Herb, e & $\mathrm{Az} ; \mathrm{Ar}$ & $\mathrm{wt}, \mathrm{rb}$ & $\mathrm{Sb}, \mathrm{Fd}$ & Sayedur73 \\
\hline $\begin{array}{l}\text { Rottboellia cochinchinensis (Lour.) } \\
\text { Clayton }\end{array}$ & Bara swati & NMS & Herb, $p$ & $\mathrm{Mz} ; \mathrm{Sr}$ & $\mathrm{gl}$ & $\mathrm{W}$ & Sayedur201 \\
\hline *Saccharum spontaneum L. & Kash & NMS & Herb, e & $\mathrm{Mz} ; \mathrm{Sr}$ & $\mathrm{gl}$ & $\mathrm{Sb}$ & Sayedur741 \\
\hline Sporobolus indicus (L.) R. Br. & Ailbela ghas & NMS & Herb, e & $\mathrm{Pz} ; \mathrm{Kh}$ & $\mathrm{gl}$ & $\mathrm{W}$ & Sayedur578 \\
\hline${ }^{*}$ S. tremulus (Willd.) Kunth & Jhola durba & NMS & Herb, $p$ & $\mathrm{Mz} ; \mathrm{Sr}$ & wt & $\mathrm{AW}$ & N1196 \\
\hline Themeda intermedia (Hack.) Bor & Medimedaghas & NMS & Herb, e & $\mathrm{Oz}, \mathrm{Mz} ; \mathrm{Sr}$ & wt & $\mathrm{Fd}$ & Sayedur277 \\
\hline${ }^{*}$ Zoysia matrella (L.) Merr. & Baissa ghas & MAS & Herb, $p$ & $\mathrm{Az} ; \mathrm{Ar}$ & $\mathrm{gl}$, wt & $\mathrm{Sb}, \mathrm{Fd}$ & Sayedur56 \\
\hline
\end{tabular}


Table 2. Contd.

\begin{tabular}{|c|c|c|c|c|c|c|c|}
\hline Scientific name & Bangla name & $\mathrm{MC}$ & Habit & Distribution & Habitat & Use & RS \\
\hline \multicolumn{8}{|l|}{ Typhaceae Juss. } \\
\hline $\begin{array}{l}\text { *Typha domingensis (Pers.) Poir. ex } \\
\text { Steud. }\end{array}$ & Hogla & MAS & Herb, e & $\mathrm{Oz} ; \mathrm{Sr}$ & wt & Mt & Sayedur748 \\
\hline \multicolumn{8}{|l|}{ Zingiberaceae Martinov } \\
\hline${ }^{*}$ Alpinia nigra (Gaertn.) B.L. Burtt & Tara & NMS & Herb, e & $\mathrm{Oz} ; \mathrm{Sr}$ & $\mathrm{wt}, \mathrm{rb}$ & M & Sayedur2219 \\
\hline Curcuma longa L. & Haldi & NMS & Herb, e & $\mathrm{Az} ; \mathrm{Ar}$ & $\mathrm{ml}(\mathrm{pl})$ & $\mathrm{Sp}, \mathrm{M}$ & Sayedur 573 \\
\hline \multicolumn{8}{|l|}{ Cannaceae Juss. } \\
\hline Canna indica $\mathrm{L}$. & Kolabati & NMS & Herb, e & $\mathrm{Pz} ; \mathrm{Kh}$ & $\mathrm{ml}$ & $\mathrm{O}, \mathrm{M}$ & Sayedur1150 \\
\hline \multicolumn{8}{|l|}{ Pontederiaceae Kunth } \\
\hline Eichhornia crassipes (Mart.) Solms & Kachoripana & NMS & Herb, a & $\mathrm{Oz} ; \mathrm{Sr}$ & wt & $\mathrm{Fd}, \mathrm{Mn}$ & Sayedur1014 \\
\hline Monochoria hastata (L.) Solms & Baranukha & NMS & Herb, a & $\mathrm{Mz} ; \mathrm{Sr}$ & wt & $\mathrm{Fd}$ & Sayedur265 \\
\hline $\begin{array}{l}\text { M. vaginalis (Burm. f.) C. Presl ex } \\
\text { Kunth }\end{array}$ & Nukha & NMS & Herb, a & $\mathrm{Mz} ; \mathrm{Sr}$ & wt & $\mathrm{Fd}$ & Sayedur211 \\
\hline \multicolumn{8}{|l|}{ Liliaceae Juss. } \\
\hline${ }^{*}$ Crinum asiaticum L. & Bara kanur & MAS & Herb, e & $\mathrm{Az} ; \mathrm{Ar}$ & $\mathrm{ml}$ & $\mathrm{O}, \mathrm{M}$ & Sayedur 252 \\
\hline C. defixum Ker Gawl & Sukhdarshan & NMS & Herb, e & $\mathrm{Oz}, \mathrm{Mz} ; \mathrm{Sr}, \mathrm{Kh}$ & $\mathrm{wt}, \mathrm{rb}$ & $\mathrm{O}, \mathrm{M}$ & Sayedur363 \\
\hline C. latifolium L. & Sukhdarshan & NMS & Herb, e & $\mathrm{Mz} ; \mathrm{Sr}$ & $\mathrm{ml}$ & $\mathrm{O}$ & Sayedur241 \\
\hline Curculigo orchioides Gaertn. & Talmuli & NMS & Herb, e & $\mathrm{Mz} ; \mathrm{Sr}$ & $\mathrm{ml}$ & M & Sayedur157 \\
\hline \multicolumn{8}{|l|}{ Smilacaceae Vent. } \\
\hline Smilax ovalifolia Roxb. & Kumarilata & NMS & Herb, c & $\mathrm{Oz} ; \mathrm{Sr}$ & $\mathrm{fm}$ & M & Sayedur713 \\
\hline \multicolumn{8}{|l|}{ Dioscoreaceae R. Br. } \\
\hline Dioscorea bulbifera L. & Mou alo & NMS & Herb, c & $\mathrm{Pz} ; \mathrm{Kh}$ & $\mathrm{fm}$, os & $\mathrm{M}, \mathrm{V}$ & Sayedur1154 \\
\hline \multicolumn{8}{|l|}{ Orchidaceae Juss. } \\
\hline${ }^{*}$ Acampe ochracea (Lindl.) Hochr. & Kampera & NMS & Herb, ep & $\mathrm{Mz}, \mathrm{Pz} ; \mathrm{Ch}, \mathrm{Kh}$ & $\mathrm{tt}$ & $\mathrm{O}$ & Sayedurb352 \\
\hline${ }^{*}$ Dendrobium anceps Sw. & Ansirium & NMS & Herb, ep & $\mathrm{Oz} ; \mathrm{Sr}$ & $\mathrm{tt}$ & $\mathrm{O}, \mathrm{M}$ & Sayedur723 \\
\hline Geodorum densiflorum (Lam.) Schltr. & Bhuiphul & NMS & Herb, e & $\mathrm{Mz} ; \mathrm{Sr}$ & $\mathrm{ml}$ & $\mathrm{O}$ & Sayedur239 \\
\hline${ }^{*}$ Luisia brachystachys (Lindl.) Blume & Brac luci & NMS & Herb, ep & $\mathrm{Pz} ; \mathrm{St}$ & tt & $\mathrm{O}$ & Sayedur1452 \\
\hline${ }^{*}$ L. teretifolia Sensu Hook. f. & Lanka luci & NMS & Herb, ep & $\mathrm{Az} ; \mathrm{Ar}$ & $\mathrm{tt}$ & $\mathrm{O}$ & Sayedur224 \\
\hline *Oberonia gammiei King \& Pantl. & Gami orchid & MAS & Herb, ep & $\mathrm{Mz} ; \mathrm{Sr}$ & $\mathrm{tt}$ & $\mathrm{O}$ & Sayedur1940 \\
\hline $\begin{array}{l}{ }^{*} \text { Pelatantheria insectifera (Rchb. f.) } \\
\text { Ridl. }\end{array}$ & Rongila pata & NMS & Herb, ep & $\mathrm{Oz}, \mathrm{Mz} ; \mathrm{Sr}$ & $\mathrm{tt}$ & $\mathrm{O}$ & Sayedur2083 \\
\hline${ }^{*}$ Trias oblonga Lindl. & Trias orchid & MAS & Herb, ep & $\mathrm{Mz} ; \mathrm{Sr}, \mathrm{Ch}$ & $\mathrm{tt}$ & $\mathrm{O}$ & Sayedur2070 \\
\hline Zeuxine strateumatica (L.) Schltr. & Setguli & NMS & Herb, e & $\mathrm{Pz} ; \mathrm{Kh}$ & $\mathrm{gl}, \mathrm{ml}$ & $\mathrm{O}$ & Sayedur556 \\
\hline
\end{tabular}

\section{Acknowledgements}

The authors gratefully acknowledge the Ministry of Science and Technology, Government of the People's Republic of Bangladesh for awarding the National Science and Technology (NST) Fellowship to the first author for conducting his Ph.D. research project on the SMFB. Thanks are also due to the Chief Conservator of Forests, Conservator of Forests of Khulna Circle, Divisional Forest Officer of Sundarban East Forest Division and Sundarban West Forest Division, and all field officials of Sundarban Forest Division for their cooperation during the field surveys all over the SMFB. 


\section{References}

Ahmed, Z.U., Hassan, M.A., Begum, Z.N.T., Khondker, M., Kabir, S.M.H., Ahmad, M., Ahmed, A.T.A., Rahman, A.K.A. and Haque, E.U. (Eds) 2008-2009. Encyclopedia of Flora and Fauna of Bangladesh. Vols 6-10, 12. Asiatic Society of Bangladesh, Dhaka.

Ara, H., Khan, B. and Uddin, S.N. (Eds) 2013. Red Data Book of Vascular Plants of Bangladesh. Vol. 2. Bangladesh National Herbarium, Dhaka, Bangladesh. pp. 1-280.

Barik, J. and Chowdhury, S. 2014. True mangrove species of Sundarbans delta, West Bengal, Eastern India. Check List 10(2): 329-334.

BFD 2015. Bangladesh Forest Department. <www.bforest.gov.bd>. Retrieved on 10 May 2015.

Biswas, S.R., Choudhury, J.K., Nishat, A. and Rahman, M.M. 2007. Do invasive plants threaten the Sundarbans mangrove forest of Bangladesh? Forest Ecology and Management 245: 1-9.

Chaffey, D.R., Miller, F.R. and Sandom, J.H. 1985. A Forest Inventory of the Sundarbans, Bangladesh. Main project report No. 140. Ovearseas Development Administration (ODA), London, UK, pp. 1-196.

Chapman, V.J. 1976. Mangrove Vegetation. Vaduz, Liechtenstein: J. Cramer, pp. 1-447.

Chowdhury, Q.I. (Ed.) 2001. The State of Sundarbans. $1^{\text {st }}$ edition., Dhaka: Forum of Environmental Journalist of Bangladesh, pp. 5-16.

Cronquist, A. 1981. An Integrated System of Classification of Flowering Plants. Columbia University Press, New York, pp. 1-1262.

Das, S. and Siddiqi, N.A. 1985. The mangrove and mangrove forest of Bangladesh. Mangrove Silviculture Division Bulletin, No. 2. Bangladesh Forest Research Institute (BFRI), Chittagong, pp. 1-142.

FAO 2007. The World's Mangroves 1980-2005. A Thematic Study Prepared in the Framework of the Global Forest Resources Assessment 2005. Food and Agriculture Organization of the United Nations, Rome, Italy, pp. 1-77.

Flora of North America Editorial Committee (Eds) 1993-2014. Flora of North America North of Mexico. Vols 1-28. New York and Oxford.

Giesen, W., Wulffraat, S., Zieren, M. and Scholten, L. 2007. Mangrove Guidebook for the Southeast Asia. FAO and Wetlands International, Dharmasarn Co. Ltd., pp. 1-186.

Hooker, J.D. 1872-1897. The Flora of British India. Vols 1-7. L. Reeve \& Co., Ashford, Kent.

Hoq, M.E. 2008. Sundarbans Mangrove: Fish and Fisheries Ecology, Resources, Productivity and Management Perspectives. Graphic Media, Dhaka, Bangladesh, pp. 1-271.

Hossain, G.M., Rahman, M.S. and Khan, S.A. 2015. Species composition, richness, density and distribution of climbers in relation to salinity in Sundarbans mangrove forest of Bangladesh. International Journal of Chemical, Environmental \& Biological Sciences (IJCEBS) 3(1): 78-83.

Hussain, M.Z. (Ed.) 2014. Bangladesh Sundarban Delta Vision 2050: A First Step in its Formulation Document 2: A Compilation of Background Information. IUCN, International Union for Conservation of Nature, Bangladesh Country Office, Dhaka, Bangladesh, pp. 1-192.

Hussain, Z. and Acharya, G. (Eds) 1994. Mangroves of the Sundarbans. Vol. 2: Bangladesh. IUCN, Bangkok, Thailand, pp. 1-257.

Hyland, B.P.M. 1972. A technique for collecting botanical specimens in rain forest. Flora Malesiana Bulletin 26: $2038-2040$.

Iftekhar, S.M. and Islam, R.M. 2004. Degradation of Bangladesh Sundarbans: A management issue. Int. Forestry Rev. 6(4): 123-135.

IPNI 2008. The International Plant Names Index. <www.ipni.org>. Retrieved on 10 May 2015.

Islam, S., Rahman, M. and Chakma, S. 2014. Plant diversity and forest structure of the three protected areas (Wildlife Sanctuaries) of Bangladesh Sundarbans: current status and management strategies. In: Hanum, I.F., Latif, A., Hakeem, K.R. and Ozturk, M. (Eds), Mangrove Ecosystem of Asia, Status, Challenges and Management Strategies. Springer New York Heidelberg Dordrecht London, pp. 127-152.

Jain, S.K. and Raw, R.R. 1977. A Handbook of Field and Herbarium Methods. Today and Tomorrows Printers and Publishers, pp. 1-157. 
Karim, A. 1988. Environmental factors and distributions of mangroves in Sundarbans with special reference to Heritiera fomes Buch. Ham. Ph.D. thesis at University of Calcutta, India.

Karim, A. 1994. Vegetation. In: Hussain, Z. and Acharya, G. (Eds), Mangroves of the Sundarbans. Vol. 2: Bangladesh. IUCN, Bangkok, Thailand, pp. 43-75.

Katebi, M.N.A. 2001. Sundarbans and forestry. In: Haider, M. (Ed.), Cyclone' 91- An Environmental and Perceptional Study. BCAS, Dhaka, pp. 79-100.

Mittermeier, R.A., Myers, N., Thomsen, J.B., Da Fonseca, G.A. and Olivieri, S. 1998. Biodiversity hotspots and major tropical wilderness areas: approaches to setting conservation priorities. Conservation Biology 12: $516-520$.

Nasir, E. and Ali, S.I. (Eds) 1980-2005. Flora of Pakistan. Vols 1-209. University of Karachi, Karachi, Pakistan.

Nishorgo 2008. Nishorgo: Bangladesh's Protected Area Management Program. <www.nishorgo.org>. Nishorgo, Bangladesh Forest Department, Dhaka, Bangladesh. Retrieved on 10 May 2015.

Pichi, S.R.E.G. 1977. Tentamen pteridophytorum genera in taxonomicum ordinem redigendi. Webbia 31: $313-512$.

Prain, D. 1903. Flora of Sundribuns. Rec. Bot. Surv. Ind. 2(4): 231-270.

Rahman, M.S., Hossain, G.M., Khan, S.A. and Uddin, S.N. 2012. Study on taxonomy and distribution of Dolichandrone spathacea (L.f.) Seem. (Fam. Bignoniaceae) - A newly recorded threatened mangrove associate species of Sundarbans. Jahangirnagar University J. Biol. Sc. 1(2): 67-76.

Rahman, M.S., Hossain, G.M., Khan, S.A. and Uddin, S.N. 2014. Colubrina javanica Miq. (Rhamnaceae) - A new angiosperm record for Bangladesh. Bangladesh J. Plant Taxon. 21(1): 199-202.

Rashid, S.H., Böcker, R., Hossain, A.B.M.E. and Khan, S.A. 2008. Undergrowth species diversity of Sundarban mangrove forest (Bangladesh) in relation to salinity. Ber. Inst. Landschafts- Pflanzenökologie Univ. Hohenheim Heft. 17: 41-56.

Siddiqui, K.U., Islam, M.A., Ahmed, Z.U., Begum, Z.N.T., Hassan, M.A., Khondker, M., Rahman, M.M., Kabir, S.M.H., Ahmed, A.T.A., Rahman, A.K.A. and Haque, E.U. (Eds) 2007 \& 2008. Encyclopedia of Flora and Fauna of Bangladesh. Vols 5 \& 11. Asiatic Society of Bangladesh, Dhaka, Bangladesh.

TROPICOS 2010. Missouri Botanical Garden. <www.tropicos.org>. Missouri Botanical Garden, Saint Louis, Missouri, USA. Retrieved on 10 May 2015.

Uddin, M.Z. and Hassan, M.A. 2004. Flora of Rema-Kalenga Wildlife Sanctuary. IUCN Bangladesh Country Office, Dhaka, Bangladesh, pp. 1-120.

Uddin, S.N., Khan, M.S., Hassan, M.A. and Alam, M.K. 1998. An annotated checklist of angiospermic flora of Sitapahar at Kaptai in Bangladesh. Bangladesh J. Plant Taxon. 5(1): 13-46.

Watson, M.F., Akiyama, S., Ikeda, H., Pendry, C.A., Rajbhandari, K.R. and Shrestha, K.K. (Eds) 2011. Flora of Nepal. Vol. 3. Magnoliaceae to Rosaceae. Royal Botanic Gardens, Edinburgh, pp. 1-512.

Wu, C.Y., Raven, P.H. and Hong, D.Y. (Eds) 1995-2013. Flora of China. Vols 2-25. Science Press and Missouri Botanical Garden Press, Beijing and St. Louis.

(Manuscript received on 5 March 2015; revised on 10 May 2015) 